

\title{
Representations up to homotopy of Lie algebroids
}

Abad, C A ; Crainic, M

\begin{abstract}
We introduce and study the notion of representation up to homotopy of a Lie algebroid, paying special attention to examples. We use representations up to homotopy to define the adjoint representation of a Lie algebroid and show that the resulting cohomology controls the deformations of the structure. The Weil algebra of a Lie algebroid is defined and shown to coincide with Kalkman's BRST model for equivariant cohomology in the case of group actions. The relation of this algebra with the integration of Poisson and Dirac structures is explained in [Arias Abad, Crainic, Ann. Inst. Fourier].
\end{abstract}

DOI: https://doi.org/10.1515/crelle.2011.095

Posted at the Zurich Open Repository and Archive, University of Zurich

ZORA URL: https://doi.org/10.5167/uzh-58213

Journal Article

Accepted Version

Originally published at:

Abad, C A; Crainic, M (2012). Representations up to homotopy of Lie algebroids. Journal für die Reine und Angewandte Mathematik, 2012(663):91-126.

DOI: https://doi.org/10.1515/crelle.2011.095 


\title{
Representations up to homotopy of Lie algebroids
}

\author{
Camilo Arias Abad and Marius Crainic \\ Department of Mathematics \\ Utrecht University \\ Utrecht, The Netherlands
}

December 7, 2010

\begin{abstract}
This is the first in a series of papers devoted to the study of the cohomology of classifying spaces. The aim of this paper is to introduce and study the notion of representation up to homotopy and to make sense of the adjoint representation of a Lie algebroid. Our construction is inspired by Quillen's notion of superconnection and fits into the general theory of structures up to homotopy. The advantage of considering such representations is that they are flexible and general enough to contain interesting examples which are the correct generalization of the corresponding notions for Lie algebras. They also allow one to identify seemingly ad-hoc constructions and cohomology theories as instances of the cohomology with coefficients in representations (up to homotopy). In particular, we show that the adjoint representation of a Lie algebroid makes sense as a representation up to homotopy and that, similar to the case of Lie algebras, the resulting cohomology controls the deformations of the Lie algebroid (i.e. it coincides with the deformation cohomology of the algebroid). Many other examples are given. At the end, we show how representations up to homotopy can be used to construct Weil algebras of Lie algebroids.
\end{abstract}

\section{Contents}

1 Introduction $\quad 2$

2 Preliminaries 4

2.1 Representations and cohomologies . . . . . . . . . . . . . . 4

2.2 Basic connections and the basic curvature . . . . . . . . . . . . 7

2.3 The graded setting . . . . . . . . . . . . . . . . . . . . . . 10

2.4 Complexes of vector bundles . . . . . . . . . . . . . . . . . 12

3 Representations up to homotopy $\quad \mathbf{1 4}$

3.1 Representations up to homotopy and first examples . . . . . . . . . . . . . . . 14

3.2 Operations and more examples . . . . . . . . . . . . . . . 21

3.3 Cohomology, the derived category, and some more examples . . . . . . . . . 24

4 The Weil algebra and the BRST model for equivariant cohomology 28

4.1 The connection dependent model . . . . . . . . . . . . . . . . . . 29

4.2 The intrinsic model . . . . . . . . . . . . . . . . . . . . . . . . . 31 


\section{Introduction}

Lie algebroids are infinite dimensional Lie algebras which can be thought of as a generalized tangent bundles associated to various geometric situations. Apart from Lie algebras and (tangent bundles of) manifolds, examples of Lie algebroids come from foliation theory, equivariant geometry, Poisson geometry, riemannian foliations, quantization, etc. Lie algebroids are the infinitesimal counterparts of Lie groupoids exactly in the same sense in which Lie algebras are related to Lie groups, and much of the classical correspondence holds in this general setting. A representation of a Lie algebroid on a vector bundle is an action by derivations on the space of sections. In the case of tangent bundles, a representation corresponds to a flat vector bundle- note that flat vector bundles on manifolds serve as coefficient systems for cohomology theories, just like representations of Lie algebras do.

The aim of this paper is to introduce and study a more general notion of representation, called representation up to homotopy. Our construction is inspired by Quillen's notion of superconnection [25] and fits into the general theory of structures up to homotopy. The idea of representations up to homotopy is to represent Lie algebroids in cochain complexes of vector bundles, rather than in vector bundles. We also allow the action to be flat only up to homotopy, that is, the curvature of the connection may not be zero, but it is controlled by some coherent homotopies which are part of the structure of the representation. The advantage of considering these representations is that they are flexible and general enough to contain interesting examples which are the correct generalization of the corresponding notions for Lie algebras. They also allow one to identify seemingly ad-hoc constructions and cohomology theories as instances of the cohomology with coefficients in representations. Our study of representations up to homotopy is motivated by applications to the following problems:

1. Cohomology of $B G$ : This is the first in a series of papers devoted to the problem of finding models for the cohomology of the classifying space $B G$ of a Lie groupoid $G$, generalizing the standard models for equivariant cohomology. The classifying space arises as the base of a universal principal $G$-bundle $E G \rightarrow B G$, which is typically infinite dimensional. When $G$ is a Lie group, the standard model for $\Omega(E G)$ is the Weil algebra $W(\mathfrak{g})$ of the Lie algebra $\mathfrak{g}$ of $G . W(\mathfrak{g})$ is built out of representations of $\mathfrak{g}$ and it contains enough information to compute the cohomology of $B G$, at least when $G$ is compact. We use representations up to homotopy to construct the Weil algebra $W(A)$ of a general Lie algebroid $A$ and prove that it is a model for the cohomology of $E G$ (if $A$ is the Lie algebroid of $G$ ). For a Lie group $G$ acting on a manifold $M$, there is an associated groupoid $\mathcal{G}=G \ltimes M$ and the cohomology of $B \mathcal{G}$ is precisely the equivariant cohomology of $M$. As we shall see, in this case the Weil algebra $W(\mathcal{A})$ is Kalkman's BRST algebra for equivariant cohomology. There is a second way in which representations up to homotopy are related to the cohomology of $B G$. The corresponding notion of representation up to homotopy of a Lie groupoid allows one to generalize Bott's spectral sequence [5] and explain the relation with the models for equivariant cohomology of Getzler [15] and Cartan [8]. This is the subject of our coming paper [2].

2. The adjoint representation: Ordinary representations are too restrictive to allow for a good notion of adjoint representation of a Lie algebroid, see example 2.6. We prove that, using representations up to homotopy, a Lie algebroid has an adjoint representation with the same formal properties as that of a Lie algebra. For instance, we will see that the associated cohomology coincides with the deformation cohomology studied in [12], and thus controls the deformation of the structure. This is a generalization of the well known result for Lie algebras.

3. Cyclic cohomology: Lie groupoids, via their convolution algebras, are the main source of examples of noncommutative spaces and provide a link between classical and non- 
commutative geometry. The cyclic cohomology of such convolution algebras appears as "the correct De-Rham cohomology" in the non-commutative context. The computation of this cohomology for general Lie groupoids is a wide open problem, and one of the reasons is the lack of a representation theory which is general enough to handle the objects arising in the calculations. We believe that the notion of representation up to homotopy of Lie algebroids and of Lie groupoids (to be treated in [2]) may help deal with this issue.

4. Cocycle equations: One of the standard ways of understanding and handling complicated equations is to interpret them as cocycle equations and treat them as part of a cohomology theory. Sometimes it is not clear what the relevant cohomology theory is -for example when looking at the infinitesimal equations underlying multiplicative two forms (see the equations in the main theorem of [7]), or the equations satisfied by the $k$-differentials of [18]. Representations up to homotopy and the associated cohomologies provide a framework in which one can give a cohomological interpretation to various otherwise strange looking equations. There is a significant conceptual and practical advantage in this point of view. For instance, the infinitesimal equations associated to multiplicative two forms can be seen as cocycle equations in the Weil algebra (Proposition 4.8) and the k-differentials are cocycle equations for other representations (Proposition 3.27). With this in mind, one can recover the main result of [7] on the integration of Dirac structures as a consequence of a Van Est isomorphism theorem- this new proof is essentially different from the original one and will be presented in [3].

Let us now mention how this paper intersects related work that we have found in the literature. The question of constructing the adjoint representation of a Lie algebroid has already been addressed by Evens, Lu and Weinstein in [14]. Even though the two points of view are obviously related, we stress that our notion of representation up to homotopy differs fundamentally from the one used in their paper. Our Weil algebra is probably isomorphic to the one given by Mehta in his thesis [24], where, using the language of supermanifolds, he described the algebra $\left(C^{\infty}([-1] T([-1] A)), \mathcal{L}_{d_{A}}+d\right)$. Similar descriptions were communicated to us by D. Roytenberg and P. Severa (unpublished). Some of the equations that appear in the definition of the adjoint representation where considered by Blaom in [4], which appeared while this work was being done.

This paper is organized as follows. Section 2 begins by collecting the known definitions of Lie algebroids, representations and cohomology theories. Then, the connections and curvatures underlying the adjoint representation are described and given a geometric interpretation. Finally, to fix our conventions, we recall some basic properties of graded algebra and complexes of vector bundles.

In Section 3 we give the definition of representations up to homotopy and introduce several examples, including the adjoint representation. We show that this notion has the usual properties of structures up to homotopy (Proposition 3.37 and Theorem 3.38). We study how to compute the associated cohomology and derive some invariance properties (Proposition 3.34). In particular, we prove that the cohomology associated to the adjoint representation is isomorphic to the deformation cohomology of [12] (Theorem 3.10). We explain the relation between extensions of Lie algebroids and representations up to homotopy (Proposition 3.14). The parallel transport arising from representations up to homotopy is explained in the case of tangent bundles (Proposition 3.19).

In Section 4 we discuss several isomorphic models for the Weil algebra of a Lie algebroidgeneralizing the standard Weil algebra of a Lie algebra. We show that, when applying this construction to the Lie algebroid associated to a Lie group action on a manifold, one obtains Kalkman's BRST algebra for equivariant cohomology (Proposition 4.5). 


\section{Preliminaries}

\subsection{Representations and cohomologies}

In this subsection we review known facts about Lie algebroids, representations and cohomology. We recall some cocycle equations one finds in the literature (Remark 2.7) and make general comments regarding the notion of adjoint representation for Lie algebroids.

Throughout this paper, $A$ denotes a Lie algebroid over a fixed base manifold $M$. As a general reference for algebroids, we use [22].

Definition 2.1. A Lie algebroid over $M$ is a vector bundle $\pi: A \rightarrow M$ together with a bundle map $\rho: A \rightarrow T M$, called the anchor map, and a Lie bracket in the space $\Gamma(A)$ of sections of A satisfying Leibnitz identity:

$$
[\alpha, f \beta]=f[\alpha, \beta]+\rho(\alpha)(f) \beta
$$

for every $\alpha, \beta \in \Gamma(A)$ and $f \in C^{\infty}(M)$.

Given an algebroid $A$, there is an associated De-Rham complex $\Omega(A)=\Gamma\left(\Lambda A^{*}\right)$, with the De-Rham operator given by the Koszul formula

$$
\begin{aligned}
d_{A} \omega\left(\alpha_{1}, \ldots, \alpha_{n+1}\right)= & \sum_{i<j}(-1)^{i+j} \omega\left(\left[\alpha_{i}, \alpha_{j}\right], \ldots, \hat{\alpha_{i}}, \ldots, \hat{\alpha_{j}}, \ldots, \alpha_{k+1}\right) \\
& +\sum_{i}(-1)^{i+1} L_{\rho\left(\alpha_{i}\right)} \omega\left(\alpha_{1}, \ldots, \hat{\alpha_{i}}, \ldots, \alpha_{k+1}\right)
\end{aligned}
$$

where $L_{X}(f)=X(f)$ is the Lie derivative along vector fields. The operator $d_{A}$ is a differential $\left(d_{A}^{2}=0\right)$ and satisfies the derivation rule

$$
d_{A}(\omega \eta)=d_{A}(\omega) \eta+(-1)^{p} \omega d_{A}(\eta),
$$

for all $\omega \in \Omega^{p}(A), \eta \in \Omega^{q}(A)$.

Definition 2.2. Let $A$ be a Lie algebroid over $M$. An A-connection on a vector bundle $E$ over $M$ is a bilinear map $\nabla: \Gamma(A) \times \Gamma(E) \rightarrow \Gamma(E),(\alpha, S) \mapsto \nabla_{\alpha}(S)$ such that:

$$
\nabla_{f \alpha}(s)=f \nabla_{\alpha}(s), \quad \nabla_{\alpha}(f s)=f \nabla_{\alpha}(s)+L_{\rho(\alpha)}(f)(s)
$$

for all $f \in C^{\infty}(M), s \in \Gamma(E)$ and $\alpha \in \Gamma(A)$. The A-curvature of $\nabla$ is the tensor given by

$$
R_{\nabla}(\alpha, \beta)(s):=\nabla_{\alpha} \nabla_{\beta}(s)-\nabla_{\beta} \nabla_{\alpha}(s)-\nabla_{[\alpha, \beta]}(s)
$$

for $\alpha, \beta \in \Gamma(A), s \in \Gamma(E)$. The A-connection $\nabla$ is called flat if $R_{\nabla}=0$. A representation of $A$ is a vector bundle $E$ together with a flat $A$-connection $\nabla$ on $E$.

Given any $A$-connection $\nabla$ on $E$, the space of $E$-valued $A$-differential forms, $\Omega(A ; E)=$ $\Gamma\left(\Lambda A^{*} \otimes E\right)$ has an induced operator $d_{\nabla}$ given by the Koszul formula

$$
\begin{aligned}
d_{\nabla} \omega\left(\alpha_{1}, \ldots, \alpha_{k+1}\right) & =\sum_{i<j}(-1)^{i+j} \omega\left(\left[\alpha_{i}, \alpha_{j}\right], \ldots, \hat{\alpha_{i}}, \ldots, \hat{\alpha_{j}}, \ldots, \alpha_{k+1}\right)+ \\
& +\sum_{i}(-1)^{i+1} \nabla_{\alpha_{i}} \omega\left(\alpha_{1}, \ldots, \hat{\alpha_{i}}, \ldots, \alpha_{k+1}\right) .
\end{aligned}
$$

In general, $d_{\nabla}$ satisfies the derivation rule

$$
d_{\nabla}(\omega \eta)=d_{A}(\omega) \eta+(-1)^{p} \omega d_{\nabla}(\eta),
$$

but squares to zero if and only if $\nabla$ is flat. 
Proposition 2.3. Given a Lie algebroid $A$ and a vector bundle $E$ over $M$, there is a 1-1 correspondence between $A$-connections $\nabla$ on $E$ and degree +1 operators $d_{\nabla}$ on $\Omega(A ; E)$ which satisfy the derivation identity. Moreover, $(E, \nabla)$ is a representation if and only if $d_{\nabla}^{2}=0$.

In a more algebraic language, every Lie algebroid $A$ has an associated DG algebra $\left(\Omega(A), d_{A}\right)$, and every representation $E$ of $A$ gives a DG module over this DG algebra.

Definition 2.4. Given a representation $E=(E, \nabla)$ of $A$, the cohomology of $A$ with coeffcients in $E$, denoted $H^{\bullet}(A ; E)$, is the cohomology of the complex $\left(\Omega(A ; E), d_{\nabla}\right)$. When $E$ is the trivial representation (the trivial line bundle with $\left.\nabla_{\alpha}=L_{\rho(\alpha)}\right)$ ), we write $H^{\bullet}(A)$.

The deformation cohomology of $A$ arises in the study of the deformations of the Lie algebroid structure [12]. This cohomology cannot, in general, be realized as the cohomology associated to any representation. The deformation complex $\left(C_{\mathrm{def}}^{\bullet}(A), \delta\right)$ is defined as follows. In degree $k$, it consists of antisymmetric, $\mathbb{R}$-multilinear maps

$$
c: \underbrace{\Gamma(A) \times \cdots \times \Gamma(A)}_{k-\text { times }} \rightarrow \Gamma(A)
$$

which are multiderivations, in the sense that they come with a multilinear map, the symbol of $c$ :

$$
\sigma_{c}: \underbrace{\Gamma(A) \times \cdots \times \Gamma(A)}_{k-1-\text { times }} \rightarrow \Gamma(T M)
$$

satisfying:

$$
c\left(\alpha_{1}, \ldots, f \alpha_{k}\right)=f c\left(\alpha_{1}, \ldots, \alpha_{k}\right)+L_{\sigma_{c}\left(\alpha_{1}, \ldots, \alpha_{k-1}\right)}(f) \alpha_{k},
$$

for any function $f \in C^{\infty}(M)$ and sections $\alpha_{i} \in \Gamma(A)$. The differential

$$
\delta: C_{\mathrm{def}}^{k}(A) \rightarrow C_{\mathrm{def}}^{k+1}(A)
$$

is given by

$$
\begin{aligned}
\delta(c)\left(\alpha_{1}, \ldots, \alpha_{k+1}\right)= & \sum_{i<j}(-1)^{i+j} c\left(\left[\alpha_{i}, \alpha_{j}\right], \alpha_{1}, \ldots, \hat{\alpha_{i}}, \ldots, \hat{\alpha_{j}}, \ldots, \alpha_{k+1}\right) \\
& +\sum_{i=1}^{k+1}(-1)^{i+1}\left[\alpha_{i}, c\left(\alpha_{1}, \ldots, \hat{\alpha_{i}}, \ldots, \alpha_{k+1}\right)\right] .
\end{aligned}
$$

Definition 2.5. The deformation cohomology of the Lie algebroid $A$, denoted $H_{\text {def }}^{\bullet}(A)$ is defined as the cohomology of the cochain complex $\left(C_{d e f}^{\bullet}(A), \delta\right)$.

\section{Example 2.6.}

1. In the extreme case when $A$ is $T M$, its representations are the flat vector bundles over $M$, while the associated cohomology is the usual cohomology of $M$ with local coefficients given by the flat sections of the vector bundle. The deformation cohomology is trivial in this case (cf. [12], Corollary 2).

2. When $A=\mathfrak{g}$ is a Lie algebra (hence $M$ is a point), one recovers the standard notion of representation of Lie algebras, and Lie algebra cohomology. The deformation cohomology in this case is isomorphic to $H^{\bullet}(\mathfrak{g} ; \mathfrak{g})$ - the cohomology with coefficients in the adjoint representation. This is related to the fact that deformations of the Lie algebra $\mathfrak{g}$ are controlled by $H^{2}(\mathfrak{g} ; \mathfrak{g})$.

3. When $A=\mathcal{F}$ is a foliation on $M$, viewed as an involutive sub-bundle of $T M$, the Lie algebroid cohomology becomes the well-known foliated cohomology (see e.g. [1, 20]). Similar to the adjoint representation in the case of Lie algebras, there is a canonical 
non-trivial representation of $\mathcal{F}$ : it is the normal bundle $\nu=T M / \mathcal{F}$, endowed with the Bott connection [6]

$$
\nabla_{V}(X \bmod \mathcal{F})=[V, X] \bmod \mathcal{F} .
$$

The resulting cohomology $H^{\bullet}(\mathcal{F} ; \nu)$ was introduced by Heitsch in his study of deformations of foliations [16], where he explains that such deformations are controlled by $H^{1}(\mathcal{F} ; \nu)$. This is just a first indication of the fact that, for foliations, $H_{\text {def }}^{\bullet}(\mathcal{F})$ is isomorphic to $H^{\bullet-1}(\mathcal{F} ; \nu)$ (Proposition 4 in [12]). To summarize, $\nu$ plays the role of the adjoint representation of Lie algebras, modulo a shift by one in the degree. In other words, we may declare the adjoint representation of $\mathcal{F}$ to be $\nu[-1]$ - the graded vector bundle which is $\nu$ concentrated in degree one.

4. If $A$ is a regular Lie algebroid, i.e. if $\rho: A \rightarrow T M$ has constant rank, then $A$ has two canonical representations. They are the kernel of $\rho$, denoted $\mathfrak{g}(A)$, with the $A$ connection

$$
\nabla_{\alpha}^{\operatorname{adj}}(\beta)=[\alpha, \beta]
$$

and the normal bundle $\nu(A)=T M / \rho(A)$ of the foliation induced by $A$, with the Bott-like connection

$$
\nabla_{\alpha}^{\operatorname{adj}}(\bar{X})=\overline{[\rho(\alpha), X]}
$$

where $\bar{X}=X \bmod \rho(A)$. It is tempting to define the adjoint representation of $A$ to be the sum of these two representations, viewed as a graded vector bundle with $\mathfrak{g}(A)$ in degree zero and $\nu(A)$ in degree one. Is it reasonable to define

$$
\operatorname{Ad}=\mathfrak{g}(A) \oplus \nu(A)[-1] ?
$$

The situation is a bit subtle. The sum of the cohomologies associated to these two representations is not necessarily isomorphic to the deformation cohomology $H_{\text {def }}^{\bullet}(A)$. Instead, there is a long exact sequence (Theorem 3 in [12]) relating these groups:

$$
\cdots \rightarrow H^{n}(A ; \mathfrak{g}(A)) \rightarrow H_{\mathrm{def}}^{n}(A) \rightarrow H^{n-1}(A ; \nu(A)) \stackrel{\delta}{\rightarrow} H^{n+1}(A ; \mathfrak{g}(A)) \rightarrow \cdots
$$

and the connecting map $\delta$ may be non-zero. As we shall see, definition (1) is correct provided we endow the objects involved with the right structure (and not just that of representations).

5. For general Lie algebroids, there may be disappointingly few representations. In particular, the representations $\mathfrak{g}(A)$ and $\nu(A)$ mentioned above no longer exist (they are not, in general, vector bundles). Hence the situation seems even worse when it comes to looking for the adjoint representation. However, the graded direct sum $\mathfrak{g}(A) \oplus \nu(A)[1]$ (which should be part of the adjoint representation, but which is non-smooth in general) can be viewed as the cohomology of a cochain complex of vector bundles, concentrated in two degrees:

$$
A \stackrel{\rho}{\rightarrow} T M .
$$

We will call this the adjoint complex of $A$. The idea of using this complex in order to make sense of the adjoint representation appeared already in [14], and is also present in [11]. In this paper we work with cochain complexes of vector bundles as a replacement for their (possibly non-smooth) cohomology bundles. It is remarkable that this point of view reveals the structure missing in order to make equation (1) precise.

Remark 2.7 (cocycle-like equations). In the literature one often encounters equations which look like cocycle conditions, but which do not seem to have a cohomology theory behind them. Such equations arise naturally from various contexts (e.g. as infinitesimal manifestations of properties of interesting global objects) and it is often worth interpreting them as part of cohomology theories. This not only gives a conceptual point of view on the 
equations, but also allows one to handle them using powerful tools. We point out two such equations which will be shown later to fit perfectly into our theory.

The first one is related to the notion of $k$-differential studied in [18]. An almost $k$ differential on a Lie algebroid $A$ is a pair of linear maps $\delta: C^{\infty}(M) \rightarrow \Gamma\left(\Lambda^{k-1} A\right), \delta$ : $\Gamma(A) \rightarrow \Gamma\left(\Lambda^{k} A\right)$ satisfying

(i) $\delta(f g)=\delta(f) g+f \delta(g)$

(ii) $\delta(f \alpha)=\delta(f) \wedge \delta(\alpha)+f \delta(\alpha)$

for all $f, g \in C^{\infty}(M), \alpha \in \Gamma(A)$. It is called a $k$-differential if

$$
\delta[\alpha, \beta]=[\delta(\alpha), \beta]+[\alpha, \delta(\beta)]
$$

for all $\alpha, \beta \in \Gamma(A)$.

The second example arises from the notion of IM (infinitesimally multiplicative) form, which is the infinitesimal counterpart of multiplicative 2-forms on groupoids [7]. An IM form on a Lie algebroid $A$ is a bundle map $\sigma: A \rightarrow T^{*} M$ satisfying:

$$
\begin{aligned}
\langle\sigma(\alpha), \rho(\beta)\rangle & =-\langle\sigma(\beta), \rho(\alpha)\rangle, \\
\sigma([\alpha, \beta]) & =L_{\rho(\alpha)}(\sigma(\beta))-L_{\rho(\beta)}(\sigma(\alpha))+d\langle\sigma(\alpha), \rho(\beta)\rangle
\end{aligned}
$$

for all $\alpha, \beta \in \Gamma(A)$. Here $\rho$ is the anchor of $A$ and $\langle\cdot, \cdot\rangle$ denotes the usual pairing between a vector space and its dual.

\subsection{Basic connections and the basic curvature}

Keeping in mind our discussion on what the adjoint representation should be (Example 2.6), we want to extend the canonical flat $A$-connections $\nabla^{\text {adj }}$ (from $\mathfrak{g}(A)$ and $\nu(A)$ ) to $A$ and $T M$ or, even better, to the adjoint complex (2). This construction already appeared in the theory of secondary characteristic classes [11] and it was also used in [13].

Definition 2.8. Given a Lie algebroid $A$ over $M$ and a connection $\nabla$ on the vector bundle A, we define

1. The basic A-connection induced by $\nabla$ on $A$ :

$$
\nabla_{\alpha}^{b a s}(\beta)=\nabla_{\rho(\beta)}(\alpha)+[\alpha, \beta] .
$$

2. The basic A-connection induced by $\nabla$ on $T M$ :

$$
\nabla_{\alpha}^{\text {bas }}(X)=\rho\left(\nabla_{X}(\alpha)\right)+[\rho(\alpha), X] .
$$

Note that $\nabla^{\text {bas }} \circ \rho=\rho \circ \nabla^{\text {bas }}$, i.e. $\nabla^{\text {bas }}$ is an $A$-connection on the adjoint complex (2). On the other hand, the existence of a connection $\nabla$ such that $\nabla^{\text {bas }}$ is flat is a very restrictive condition on $A$. It turns out that the curvature of $\nabla^{\text {bas }}$ hides behind a more interesting tensor- and that is what we will call the basic curvature of $\nabla$.

Definition 2.9. Given a Lie algebroid $A$ over $M$ and a connection $\nabla$ on the vector bundle $A$, we define the basic curvature of $\nabla$, as the tensor

$$
R_{\nabla}^{\text {bas }} \in \Omega^{2}(A ; \operatorname{Hom}(T M, A))
$$

given by

$$
R_{\nabla}^{b a s}(\alpha, \beta)(X):=\nabla_{X}([\alpha, \beta])-\left[\nabla_{X}(\alpha), \beta\right]-\left[\alpha, \nabla_{X}(\beta)\right]-\nabla_{\nabla_{\beta}^{b a s} X}(\alpha)+\nabla_{\nabla_{\alpha}^{b a s} X}(\beta),
$$

where $\alpha, \beta$ are sections of $A$ and $X, Y$ are vector fields on $M$. 
This tensor appears when one looks at the curvatures of the $A$-connections $\nabla^{\text {bas }}$. One may think of $R_{\nabla}^{\text {bas }}$ as the expression $\nabla_{X}([\alpha, \beta])-\left[\nabla_{X}(\alpha), \beta\right]-\left[\alpha, \nabla_{X}(\beta)\right]$ which measures the derivation property of $\nabla$ with respect to $[\cdot, \cdot]$, corrected so that it becomes $C^{\infty}(M)$-linear on all arguments.

Proposition 2.10. For any connection $\nabla$ on $A$, one has:

1. The curvature of the A-connection $\nabla^{\text {bas }}$ on $A$ equals to $-\rho \circ R_{\nabla}^{b a s}$, while the curvature of the $A$-connection $\nabla^{\text {bas }}$ on TM equals to $-R_{\nabla}^{\text {bas }} \circ \rho$.

2. $R_{\nabla}^{\text {bas }}$ is closed with respect to $\nabla^{\text {bas }}$ i.e. $d_{\nabla^{\text {bas }}}\left(R^{\text {bas }}\right)=0$.

Proof. For $\alpha, \beta, \gamma \in \Gamma(A)$,

$$
\begin{aligned}
R_{\nabla}^{\mathrm{bas}}(\alpha, \beta) \rho(\gamma)= & \nabla_{\rho(\gamma)}([\alpha, \beta])-\left[\nabla_{\rho(\gamma)}(\alpha), \beta\right]-\left[\alpha, \nabla_{\rho(\gamma)}(\beta)\right] \\
& -\nabla_{\nabla_{\beta} \rho(\gamma)}(\alpha)+\nabla_{\nabla_{\alpha} \rho(\gamma)}(\beta) \\
= & \nabla_{[\alpha, \beta]}(\gamma)-\nabla_{\alpha}\left(\nabla_{\beta}(\gamma)\right)+\nabla_{\beta}\left(\nabla_{\alpha}(\gamma)\right) \\
= & -R_{\nabla_{\text {bas }}}(\alpha, \beta)(\gamma)
\end{aligned}
$$

On the other hand, if we evaluate at a vector field $X$ the computation becomes:

$$
\begin{aligned}
\rho\left(R_{\nabla}^{\mathrm{bas}}(\alpha, \beta) X\right)= & \rho\left(\nabla_{X}([\alpha, \beta])-\left[\nabla_{X}(\alpha), \beta\right]-\left[\alpha, \nabla_{X}(\beta)\right]\right. \\
& \left.-\nabla_{\nabla_{\beta} X}(\alpha)+\nabla_{\nabla_{\alpha} X}(\beta)\right) \\
= & \rho\left(\nabla_{X}([\alpha, \beta])\right)+[\rho([\alpha, \beta]), X] \\
& +[[\rho(\beta), X], \rho(\alpha)]-\rho\left(\left[\alpha, \nabla_{X}(\beta)\right]\right)-\rho\left(\nabla_{\nabla_{\beta} X}(\alpha)\right) \\
& +[\rho(\beta),[\rho(\alpha), X]]+\rho\left(\nabla_{\nabla_{\alpha} X}(\beta)\right)-\rho\left(\left[\nabla_{X}(\alpha), \beta\right]\right) \\
= & \nabla_{[\alpha, \beta]}(X)-\nabla_{\alpha}\left(\nabla_{\beta}(X)\right)+\nabla_{\beta}\left(\nabla_{\alpha}(X)\right) \\
= & -R_{\nabla_{\text {bas }}}(\alpha, \beta)(X)
\end{aligned}
$$

The proof of the second part is a similar computation that we will omit.

Let us now explain the relationship between the basic curvature $R_{\nabla}^{\text {bas }}$ and the first jet algebroid of $A$. For the first part of the discussion we only use the vector bundle structure of $A$. We denote by $J^{1}(A)$ the first jet bundle of $A$ and by $\pi: J^{1}(A) \rightarrow A$ the canonical projection. There is an extension of vector bundles over $M$,

$$
\operatorname{Hom}(T M, A) \stackrel{i}{\rightarrow} J^{1}(A) \stackrel{\pi}{\rightarrow} A
$$

where the inclusion $i$ is uniquely determined by the condition

$$
\operatorname{Hom}(T M, A) \ni d f \otimes \alpha \mapsto f j^{1}(\alpha)-j^{1}(f \alpha)
$$

for all $f \in C^{\infty}(M), \alpha \in \Gamma(A)$. Giving a connection $\nabla$ on $A$ is equivalent to choosing a splitting $j^{\nabla}: A \rightarrow J^{1}(A)$ of $\pi$. The relation between the two is given by

$$
j^{\nabla}(\alpha)=j^{1}(\alpha)+\nabla \cdot(\alpha)
$$

for all $\alpha \in \Gamma(A)$. Here $\nabla .(\alpha) \in \operatorname{Hom}(T M, A)$ is given by $X \mapsto \nabla_{X}(\alpha)$. This follows from the fact that, although $\alpha \mapsto j^{1}(\alpha)$ is not a bundle map, it defines a splitting of the sequence (3) at the level of sections. Hence any splitting $j$ of our sequence differs from $j^{1}$ (at the level of sections) by elements coming from $\operatorname{Hom}(T M, A)$. Moreover, the $C^{\infty}(M)$-linearity of $j^{\nabla}$ translates precisely into the derivation property for $\nabla$.

Let us now use the fact that $A$ is a Lie algebroid. In this case, $J^{1}(A)$ admits a unique Lie algebroid structure such that for any section $\alpha$ of $A$,

$$
\rho\left(j^{1} \alpha\right)=\rho(\alpha)
$$


and for any sections $\alpha, \beta \in \Gamma(A)$ :

$$
\left[j^{1} \alpha, j^{1} \beta\right]=j^{1}([\alpha, \beta]) .
$$

Since $\pi$ preserves the Lie bracket, it follows that $\operatorname{Hom}(T M, A)$ inherits the structure of a bundle of Lie algebras, and (3) becomes an extension of Lie algebroids.

Proposition 2.11. For any connection $\nabla$ on $A$,

$$
R_{\nabla}^{\text {bas }}(\alpha, \beta)=j^{\nabla}([\alpha, \beta])-\left[j^{\nabla}(\alpha), j^{\nabla}(\beta)\right] .
$$

Let us point out two results which indicate the geometric meaning of the basic curvature $R_{\nabla}^{\text {bas }}$. The first one refers to the characterization of Lie algebroids which arise from Lie algebra actions.

Proposition 2.12. A Lie algebroid A over a simply connected manifold $M$ is the algebroid associated to a Lie algebra action on $M$ if an only if it admits a flat connection $\nabla$ whose induced basic curvature $R_{\nabla}^{\text {bas }}$ vanishes.

Proof. If $A$ is associated to a Lie algebra action, one chooses $\nabla$ to be the obvious flat connection. Assume now that there is a connection $\nabla$ as above. Since $M$ is simply connected, the bundle is trivial. Choose a frame of flat sections $\alpha_{1}, \ldots, \alpha_{r}$ of $A$, and write

$$
\left[\alpha_{i}, \alpha_{j}\right]=\sum_{k=1}^{r} c_{i j}^{k} \alpha_{k}
$$

with $c_{i j}^{k} \in C^{\infty}(M)$. Since

$$
R_{\nabla}^{\mathrm{bas}}\left(\alpha_{i}, \alpha_{j}\right)(X)=\sum_{k=1}^{r} \nabla_{X}\left(c_{i j}^{k} \alpha_{k}\right)=\sum_{k=1}^{r} X\left(c_{i j}^{k}\right) \alpha_{k},
$$

we deduce that the $c_{i j}^{k}$ 's are constant. The Jacobi identity for the Lie bracket on $\Gamma(A)$ implies that $c_{i j}^{k}$ 's are the structure constants of a Lie algebra, call it $\mathfrak{g}$. The anchor map defines an action of $\mathfrak{g}$ on $M$, and the trivialization of $A$ induces the desired isomorphism.

In particular consider $A=T M$ for some compact simply connected manifold $M$ and a flat connection $\nabla$ on $A$. The condition that the basic curvature vanishes means precisely that the conjugated connection $\bar{\nabla}$ defined by:

$$
\bar{\nabla}_{X}(Y)=[X, Y]+\nabla_{Y}(X)
$$

is also flat. This implies that $M$ is a Lie group.

The next result refers to the relation between bundles of Lie algebras (viewed as Lie algebroids with zero anchor map) and Lie algebra bundles, for which the fiber Lie algebra is fixed in the local trivializations.

Proposition 2.13. Let $A$ be a bundle of Lie algebras over $M$. Then $A$ is a Lie algebra bundle if and only if it admits a connection $\nabla$ whose basic curvature vanishes.

Proof. If the bundle of Lie algebras is locally trivial then locally one can choose connections with zero $A$-curvature. Then one can use partitions of unity to construct a global connection with the same property. For the converse, assume there exists a $\nabla$ with $R_{\nabla}^{A}=0$, and we need to prove that the Lie algebra structure on the fiber is locally trivial. We may assume that $A=R^{n} \times R^{r}$ as a vector bundle. The vanishing of the basic curvature means that $\nabla$ acts as derivations of the Lie algebra fibers:

$$
\nabla_{X}([\alpha, \beta])=\left[\nabla_{X}(\alpha), \beta\right]+\left[\alpha, \nabla_{X}(\beta)\right]
$$

Since derivations are infinitesimal automorphisms, we deduce that the parallel transports induced by $\nabla$ are Lie algebra isomorphisms, providing the necessary Lie algebra bundle trivialization. 


\subsection{The graded setting}

We have already seen (Example 2.6) that in order to make sense of the adjoint representation of a Lie algebroid, we will have to consider graded vector bundles. In this section we collect a few constructions and conventions regarding the graded setting. As a general rule, we will be constantly using the standard sign convention: whenever two graded objects $x$ and $y$, say of degrees $p$ and $q$, are interchanged, one introduces a sign $(-1)^{p q}$. For instance, the standard commutator $x y-y x$ is replaced by the graded commutator

$$
[x, y]=x y-(-1)^{p q} y x .
$$

Throughout this section, $M$ is a fixed manifold, and all our vector bundles are over $M$.

1. Graded vector bundles. By a graded vector bundle over $M$ we mean a vector bundle $E$ together with a direct sum decomposition indexed by integers:

$$
E=\bigoplus_{n} E^{n}
$$

An element $v \in E^{n}$ is called a homogeneous element of degree $n$ and we write $|v|=n$. Most of the constructions on graded vector bundles follow by applying pointwise the standard constructions with graded vector spaces. Here are some of them.

1. Given two graded vector bundles $E$ and $F$, their direct sum and their tensor product have natural gradings. On $E \otimes F$ we always use the total grading:

$$
\operatorname{deg}(e \otimes f)=\operatorname{deg}(e)+\operatorname{deg}(f) .
$$

2. Given two graded vector bundles $E$ and $F$ one can also form the new graded space $\underline{\operatorname{Hom}}(E, F)$. Its degree $k$ part, denoted $\underline{\operatorname{Hom}}^{k}(E, F)$, consists of vector bundle maps $T: E \rightarrow F$ which increase the degree by $k$. When $E=F$, we use the notation $\underline{\operatorname{End}}(E)$.

3. For any graded vector bundle, the associated tensor algebra bundle $T(E)$ is graded by the total degree

$$
\operatorname{deg}\left(v_{1} \otimes \ldots \otimes v_{n}\right)=\operatorname{deg}\left(v_{1}\right)+\ldots+\operatorname{deg}\left(v_{n}\right) .
$$

The associated symmetric algebra bundle $S(E)$ is defined (fiberwise) as the quotient of $T(E)$ by forcing $[v, w]=0$ for all $v, w \in E$, while for the exterior algebra bundle $\Lambda(E)$ one forces the relations $v w=-(-1)^{p q} w v$ where $p$ and $q$ are the degrees of $v$ and $w$, respectively.

4. The dual $E^{*}$ of a graded vector bundle is graded by

$$
\left(E^{*}\right)^{n}=\left(E^{-n}\right)^{*} .
$$

2. Wedge products. We now discuss wedge products in the graded context. First of all, given a Lie algebroid $A$ and a graded vector bundle $E$, the space of $E$-valued $A$-differential forms, $\Omega(A ; E)$, is graded by the total degree:

$$
\Omega(A ; E)^{p}=\bigoplus_{i+j=p} \Omega^{i}\left(A ; E^{j}\right)
$$

Wedge products arise in the following general situation. Assume that $E, F$ and $G$ are graded vector bundles and

$$
h: E \otimes F \rightarrow G
$$

is a degree preserving vector bundle map. Then there is an induced wedge-product operation

$$
\Omega(A ; E) \times \Omega(A ; F) \rightarrow \Omega(A ; G),(\omega, \eta) \mapsto \omega \wedge_{h} \eta .
$$


Explicitly, for $\omega \in \Omega^{p}\left(A ; E^{i}\right), \eta \in \Omega^{q}\left(A ; F^{j}\right), \omega \wedge_{h} \eta \in \Omega^{p+q}\left(A ; G^{i+j}\right)$ is given by

$$
\left(\alpha_{1}, \ldots, \alpha_{p+q}\right) \mapsto \sum(-1)^{q i} \operatorname{sgn}(\sigma) h\left(\omega\left(\alpha_{\sigma(1)}, \ldots, \alpha_{\sigma(p)}\right), \eta\left(\alpha_{\sigma(p+1)} \ldots, \alpha_{\sigma(p+q)}\right)\right),
$$

where the sum is over all $(p-q)$-shuffles. One explanation for the sign is that it forces

$$
\omega \wedge_{h} \eta=(-1)^{\operatorname{deg}(\omega) \operatorname{deg}(\eta)} \eta \wedge_{\bar{h}} \omega,
$$

where $\bar{h}$ is the composition of $h$ with the graded twist map:

$$
F \otimes E \rightarrow E \otimes F, \quad u \otimes v \mapsto(-1)^{\operatorname{deg}(u) \operatorname{deg}(v)} v \otimes u
$$

Here is a list of the wedge products that will appear in this paper:

1. If $h$ is the identity we get:

$$
\cdot \wedge \cdot: \Omega(A ; E) \otimes \Omega(A ; F) \rightarrow \Omega(A ; E \otimes F) .
$$

In particular, we get two operations

$$
\Omega(A) \otimes \Omega(A ; E) \rightarrow \Omega(A ; E), \Omega(A ; E) \otimes \Omega(A) \rightarrow \Omega(A ; E) .
$$

which make $\Omega(A ; E)$ into a (graded) $\Omega(A)$-bimodule. Note that, while the first one coincides with the wedge product applied to $E$ viewed as a (ungraded) vector bundle, the second one involves a sign.

2. If $h$ is the composition of endomorphisms of $E$ we get an operation

$$
\cdot \text { ०. : } \Omega(A, \underline{\operatorname{End}}(E)) \otimes \Omega(A ; \underline{\operatorname{End}}(E)) \rightarrow \Omega(A, \underline{\operatorname{End}}(E))
$$

which gives $\Omega(A, \underline{\text { End }}(E))$ the structure of a graded algebra. Of course, this operation makes sense for general Hom's instead of End.

3. If $h$ is the evaluation map ev $: \underline{\operatorname{End}}(E) \otimes E \rightarrow E,(T, v) \mapsto T(v)$, we get:

$$
\cdot \wedge \cdot: \Omega(A ; \underline{\operatorname{End}}(E)) \otimes \Omega(A ; E) \rightarrow \Omega(A ; E)
$$

while when $h$ is the twisted evaluation map $\overline{\operatorname{ev}}: E \otimes \underline{\operatorname{End}}(E) \rightarrow E,(v, T) \mapsto(-1)^{|v||T|} T(v)$, we get:

$$
\cdot \wedge \cdot: \Omega(A ; E) \otimes \Omega(A ; \underline{\operatorname{End}}(E)) \rightarrow \Omega(A ; E) .
$$

These operations make $\Omega(A ; E)$ a graded $\Omega(A ; \underline{\text { End }}(E))$-bimodule.

4. If $h: \Lambda^{\bullet} E \otimes \Lambda^{\bullet} E \rightarrow \Lambda^{\bullet} E$ is the multiplication, we get

$$
\cdot \wedge \cdot: \Omega\left(A ; \Lambda^{\bullet} E\right) \otimes \Omega\left(A ; \Lambda^{\bullet} E\right) \rightarrow \Omega\left(A ; \Lambda^{\bullet} E\right)
$$

which makes $\Omega\left(A ; \Lambda^{\bullet} E\right)$ a graded algebra.

Note that the ring $\Omega(A ; \underline{\operatorname{End}}(E))$ can be identified with the space of endomorphisms of the left $\Omega(A)$-module $\Omega(A ; E)$ (in the graded sense). More precisely, we have the following simple lemma.

Lemma 2.14. There is a 1-1 correspondence between degree $n$ elements of $\Omega(A ; \underline{E n d}(E))$ and operators $F$ on $\Omega(A ; E)$ which increase the degree by $n$ and which are $\Omega(A)$-linear in the graded sense:

$$
F(\omega \wedge \eta)=(-1)^{n|\omega|} \omega \wedge F(\eta) \quad \forall \omega \in \Omega(A), \eta \in \Omega(A ; E) .
$$

Explicitly, $T \in \Omega(A ; \underline{\text { End }}(E))$ induces the operator $\hat{T}$ given by:

$$
\hat{T}(\eta)=T \wedge \eta
$$


Finally, there is one more interesting operation of type $\wedge_{h}$, namely the one where $h$ is the graded commutator

$$
h: \underline{\operatorname{End}}(E) \otimes \underline{\operatorname{End}}(E) \rightarrow \underline{\operatorname{End}}(E), \quad h(T, S)=T \circ S-(-1)^{|S||T|} S \circ T .
$$

The resulting operation

$$
\Omega(A, \underline{\operatorname{End}}(E)) \otimes \Omega(A ; \underline{\operatorname{End}}(E)) \rightarrow \Omega(A ; \underline{\operatorname{End}}(E))
$$

will be denoted by $[-,-]$. Note that:

$$
[T, S]=T \wedge S-(-1)^{|T||S|} S \wedge T
$$

\subsection{Complexes of vector bundles}

In this section we continue to construct the framework that will allow us to make sense of the adjoint representation of Lie algebroids. As we explained at the end of Example 2.6, in order to avoid working with non-smooth vector bundles, one realizes them as the cohomology of cochain complexes of (smooth) vector bundles. Here we bring together some rather standard constructions and facts about such complexes. We fix a manifold $M$, and all vector bundles will be over $M$.

Complexes. By a complex over $M$ we mean a cochain complex of vector bundles over $M$, i.e. a graded vector bundle $E$ over $M$ endowed with a degree one endomorphism $\partial$ satisfying $\partial^{2}=0$ :

$$
(E, \partial): \quad \cdots \stackrel{\partial}{\rightarrow} E^{0} \stackrel{\partial}{\rightarrow} E^{1} \stackrel{\partial}{\rightarrow} E^{2} \stackrel{\partial}{\rightarrow} \cdots
$$

We drop $\partial$ from the notation whenever there is no danger of confusion. A morphism between two complexes $E$ and $F$ over $M$ is a vector bundle map $f: E \rightarrow F$ which preserves the degree and is compatible with the differentials. We denote by $\operatorname{Hom}(E, F)$ the space of all such maps. We denote by $\underline{\mathrm{Ch}}(M)$ the resulting category of complexes over $M$.

Definition 2.15. We say that a complex $(E, \partial)$ over $M$ is regular if $\partial$ has constant rank. In this case one defines the cohomology of $E$ as the graded vector bundle over $M$ :

$$
\mathcal{H}^{\bullet}(E):=\operatorname{Ker}(\partial) / \operatorname{Im}(\partial)
$$

Remark 2.16. Note that $\mathcal{H}^{\bullet}(E)$ only makes sense (as a vector bundle) when $E$ is regular. On the other hand, one can always take the pointwise cohomology: for each $x \in M$, there is a cochain complex of vector spaces $\left(E_{x}, \partial_{x}\right)$ and one can take its cohomology $H^{\bullet}\left(E_{x}, \partial_{x}\right)$. The dimension of these spaces may vary as $x$ varies, and it is constant if and only if $E$ is regular, in which case they fit into a graded vector bundle over $M$ - and that is $\mathcal{H}^{\bullet}(E)$.

As for cochain complexes of vector spaces, we have the following terminology:

1. Given two complexes of vector bundles $E$ and $F$ and morphisms $f_{1}, f_{2}: E \rightarrow F$, a homotopy between $f$ and $g$ is a degree -1 map $h: E \rightarrow F$ satisfying

$$
h \partial+\partial h=f_{1}-f_{2} .
$$

If such an $h$ exists, we say that $f_{1}$ and $f_{2}$ are homotopic.

2. A morphisms $f: E \rightarrow F$ between two complexes of vector bundles $E$ and $F$ is called a homotopy equivalence if there exists a morphisms $g: F \rightarrow E$ such that $f \circ g$ and $g \circ f$ are homotopic to the identity maps. If such an $f$ exists, we say that $E$ and $F$ are homotopy equivalent. We say that $E$ is contractible if it is homotopy equivalent to the zero-complex or, equivalently, if there exists a homotopy between $\operatorname{Id}_{E}$ and the zero map. 
3. A morphism $f: E \rightarrow F$ between two complexes of vector bundles is called a quasiisomorphism if it induces isomorphism in the pointwise cohomologies. We say that $E$ is acyclic if it is pointwise acyclic.

Lemma 2.17. For complexes of vector bundles over $M$ :

(1) If $f: E \rightarrow F$ is a quasi-isomorphism at $x \in M$, then it is a quasi-isomorphism in a neighborhood of $x$. In particular, if a complex $E$ is exact at $x \in M$, then it is exact in a neighborhood of $x$.

(2) A morphism $f: E \rightarrow F$ is a quasi-isomorphism if and only if it is a homotopy equivalence. In particular, a complex $E$ is acyclic if and only if it is contractible.

(3) If a complex $E$ is regular then it is homotopy equivalent to its cohomology $\mathcal{H}^{\bullet}(E)$ endowed with the zero differential.

Proof. For (1) and (2), it suffices to prove the apparently weaker statements in the lemma coming after "in particular". This follows from the standard mapping complex argument: given a morphism $f$, one builds a double complex with $E$ as 0 -th row, $F$ as 1-st row, and $f$ as vertical differential. The resulting double complex $M(f)$, has the property that it is acyclic, or contractible, if and only if $f$ is a quasi-isomorphism, or a homotopy equivalence, respectively.

To prove the weaker statements of (1) and (2), we fix a complex $(E, \partial)$ and we choose a metric in each vector bundle $E^{i}$. Denote $\partial^{*}$ the adjoint of $\partial$ with respect to the chosen metric and

$$
\Delta=\partial \partial^{*}+\partial^{*} \partial
$$

the correspondent "Laplacian". It is not difficult to see that the complex $\left(E_{x}^{\bullet}, \partial\right)$ is exact if and only if $\Delta_{x}$ is an isomorphism. Since the isomorphisms form an open set in the space of linear transformations, we get $(1)$. When $(E, \partial)$ is exact, a simple computation shows that $h:=\Delta^{-1} \partial^{*}$ is a contracting homotopy for $E$, proving (2).

For (3) a linear version of Hodge decomposition gives us

$$
E=\operatorname{Ker}(\Delta) \oplus \operatorname{Im}(\partial) \oplus \operatorname{Im}\left(\partial^{*}\right)
$$

and an identification $\mathcal{H}^{\bullet}(E)=\operatorname{Ker}(\Delta)$. The resulting projection $E \rightarrow \operatorname{Ker}(\Delta)$ is a quasiisomorphism.

3. Operations. The operations with graded vector bundles discussed in the previous section extend to the setting of complexes. In other words, if $E$ and $F$ are complexes over $M$, then all the associated graded vector bundles $S(E), \Lambda(E), E^{*}, \underline{\operatorname{Hom}}(E, F), E \otimes F$, inherit an operator $\partial$ making them into complexes over $M$. The induced differentials are defined by requiring that they satisfy the (graded) derivation rule, written formally as:

$$
\partial(x y)=\partial(x) y+(-1)^{|x|} x \partial(y) .
$$

For instance, for $E \otimes F$,

$$
\partial(v \otimes w)=\partial(v) \otimes w+(-1)^{|v|} v \otimes \partial(w) .
$$

Also, for $T \in \underline{\operatorname{Hom}}(E, F)$,

$$
\partial(T(v))=\partial(T)(v)+(-1)^{|T|} T(\partial(v))
$$

in terms of graded commutators:

$$
\partial(T)=\partial \circ T-(-1)^{|T|} T \circ \partial=[\partial, T] .
$$


If $E$ is a complex over $M$, its differential $\partial$ induces a differential $\partial$ on $\Omega(A ; E)$ defined by:

$$
\partial(\eta)=\partial \wedge \eta
$$

Explicitly, for $\eta \in \Omega^{p}\left(A ; E^{k}\right), \partial(\eta) \in \Omega^{p}\left(A ; E^{k+1}\right)$ is given by

$$
\left(\alpha_{1}, \ldots, \alpha_{p}\right) \mapsto(-1)^{p} \partial\left(\eta\left(\alpha_{1}, \ldots, \alpha_{p}\right)\right) .
$$

The following simple lemma shows that the various differentials induced on $\Omega(A ; \underline{\operatorname{End}}(E))$ coincide.

Lemma 2.18. For any $T \in \Omega(A ; \underline{\operatorname{End}}(E))$,

$$
\partial(T)=[\partial, T]=\partial \wedge T-(-1)^{|T|} T \wedge \partial .
$$

Connections. Let $A$ be a Lie algebroid. An $A$-connection on a graded vector bundle $E$ is just an $A$-connection on the underlying vector bundle $E$ which preserves the grading. Equivalently, it is a family of $A$-connections, one on each $E^{n}$. If $(E, \partial)$ is a complex over $M$, an $A$-connection on $(E, \partial)$ is a graded connection $\nabla$ which is compatible with $\partial$ (i.e. $\left.\nabla_{\alpha} \partial=\partial \nabla_{\alpha}\right)$. Note that, in terms of the operators $d_{\nabla}$ and $\partial$ induced on $\Omega(A ; E)$, the compatibility of $\nabla$ and $\partial$ is equivalent to $\left[d_{\nabla}, \partial\right]=0$.

Connections on $E$ and $F$ naturally induce connections on the associated bundles $S(E)$, $\underline{\text { End }}(E), E \otimes F$, etc. The basic principle is, as before, the graded derivation rule. For instance, one has

$$
d_{\nabla}\left(\eta_{1} \wedge \eta_{2}\right)=d_{\nabla}\left(\eta_{1}\right) \wedge \eta_{2}+(-1)^{\left|\eta_{1}\right|} \eta_{1} \wedge d_{\nabla}\left(\eta_{2}\right)
$$

for all $\eta_{1} \in \Omega(A ; E), \eta_{2} \in \Omega(A ; F)$. Also, for $T \in \Omega(A ; \underline{\operatorname{End}}(E)), d_{\nabla}(T)$ is uniquely determined by

$$
d_{\nabla}(T \wedge \eta)=d_{\nabla}(T) \wedge \eta+(-1)^{|T|} T \wedge d_{\nabla}(\eta)
$$

for all $\eta \in \Omega(A ; E)$. More explicitly,

$$
d_{\nabla}(T)=\left[d_{\nabla}, T\right]
$$

Lemma 2.19. If a complex $(E, \partial)$ admits an $A$-connection then, for any leaf $L \subset M$ of $A$, $\left.E\right|_{L}$ is regular.

Proof. When $A=T M$ there is only one leaf $L=M$, and we have to prove that $E$ is regular. Since $\nabla$ is compatible with $\partial$, it follows that the parallel transport with respect to $\nabla$ commutes with $\partial$ and therefore induces isomorphisms between the pointwise cohomologies. The same argument applied to parallel transport along $A$-paths, as explained in [13], implies the general case.

\section{Representations up to homotopy}

\subsection{Representations up to homotopy and first examples}

In this section we introduce the notion of representation up to homotopy and the adjoint representation of Lie algebroids. As before, $A$ is a Lie algebroid over $M$. We start with the shortest, but less intuitive description of representations up to homotopy.

Definition 3.1. A representation up to homotopy of $A$ consists of a graded vector bundle $E$ over $M$ and an operator, called the structure operator,

$$
D: \Omega(A ; E) \rightarrow \Omega(A ; E)
$$


which increases the total degree by one and satisfies $D^{2}=0$ and the graded derivation rule:

$$
D(\omega \eta)=d_{A}(\omega) \eta+(-1)^{k} \omega D(\eta)
$$

for all $\omega \in \Omega^{k}(A), \eta \in \Omega(A ; E)$.

The cohomology of the resulting complex is denoted by $H^{\bullet}(A ; E)$.

Intuitively, a representation up to homotopy of $A$ is a complex endowed with an $A$ connection which is "flat up to homotopy". We will make this precise in what follows.

Proposition 3.2. There is a 1-1 correspondence between representations up to homotopy $(E, D)$ of $A$ and graded vector bundles $E$ over $M$ endowed with

1. A degree 1 operator $\partial$ on $E$ making $(E, \partial)$ a complex.

2. An A-connection $\nabla$ on $(E, \partial)$.

3. An End(E)-valued 2-form $\omega_{2}$ of total degree 1, i.e.

$$
\omega_{2} \in \Omega^{2}\left(A ; \underline{E n d}^{-1}(E)\right)
$$

satisfying

$$
\partial\left(\omega_{[2]}\right)+R_{\nabla}=0
$$

where $R_{\nabla}$ is the curvature of $\nabla$.

4. For each $i>2$ an End(E)-valued $i$-form $\omega_{i}$ of total degree 1, i.e.

$$
\omega_{i} \in \Omega^{i}\left(A ; \underline{E n d}^{1-i}(E)\right)
$$

satisfying

$$
\partial\left(\omega_{i}\right)+d_{\nabla}\left(\omega_{i-1}\right)+\omega_{2} \circ \omega_{i-2}+\omega_{3} \circ \omega_{i-3}+\ldots+\omega_{i-2} \circ \omega_{2}=0 .
$$

The correspondence is characterized by

$$
D(\eta)=\partial(\eta)+d_{\nabla}(\eta)+\omega_{2} \circ \eta+\omega_{3} \circ \eta+\ldots
$$

We also write

$$
D=\partial+\nabla+\omega_{2}+\omega_{3}+\ldots
$$

Proof. Due to the derivation rule and the fact that $\Omega(A ; E)$ is generated as an $\Omega(A)$-module by $\Gamma(E)$, the operator $D$ will be uniquely determined by what it does on $\Gamma(E)$. It will send each $\Gamma\left(E^{k}\right)$ into the sum

$$
\Gamma\left(E^{k+1}\right) \oplus \Omega^{1}\left(A ; E^{k}\right) \oplus \Omega^{2}\left(A ; E^{k-1}\right) \oplus \ldots,
$$

hence it will also send each $\Omega^{p}\left(A ; E^{k}\right)$ into the sum

$$
\Omega^{p}\left(A ; E^{k+1}\right) \oplus \Omega^{p+1}\left(A ; E^{k}\right) \oplus \Omega^{p+2}\left(A ; E^{k-1}\right) \oplus \ldots,
$$

and we denote by $D_{0}, D_{1}, \ldots$ the components of $D$. From the derivation rule for $D$, we deduce that each $D_{i}$ for $i \neq 1$ is a (graded) $\Omega(A)$-linear map and, by Lemma 2.14, it is the wedge product with an element in $\Omega(A ; \underline{E n d}(E))$. On the other hand, $D_{1}$ satisfies the derivation rule on each of the vector bundles $E^{k}$ and, by Proposition 2.3, it comes from $A$-connections on these bundles. The equations in the statement correspond to $D^{2}=0$.

Next, one can define the notion of morphism between representations up to homotopy. 
Definition 3.3. A morphism $\Phi: E \rightarrow F$ between two representations up to homotopy of $A$ is a degree zero linear map

$$
\Phi: \Omega(A ; E) \rightarrow \Omega(A ; F)
$$

which is $\Omega(A)$-linear and commutes with the structure differentials $D_{E}$ and $D_{F}$.

We denote by $\mathbb{R} e p^{\infty}(A)$ the resulting category, and by $\operatorname{Rep}^{\infty}(A)$ the set of isomorphism classes of representations up to homotopy of $A$.

By the same arguments as above, one gets the following description of morphisms in $\mathbb{R e p}^{\infty}(A)$. A morphisms is necessarily of type

$$
\Phi=\Phi_{0}+\Phi_{1}+\Phi_{2}+\cdots
$$

where $\Phi_{i}$ is a $\operatorname{Hom}(E, F)$-valued $i$-form on $A$ of total degree zero:

$$
\Phi_{i} \in \Omega^{i}\left(A ; \operatorname{Hom}^{-i}(E, F)\right)
$$

satisfying

$$
\partial\left(\Phi_{n}\right)+d_{\nabla}\left(\Phi^{n-1}\right)+\sum_{i+j=n, i \geq 2}\left[\omega_{i}, \Phi_{j}\right]=0 .
$$

Note that, in particular, $\Phi_{0}$ must be a map of complexes.

Example 3.4 (Usual representations). Of course, any representation $E$ of $A$ can be seen as a representation up to homotopy concentrated in degree zero. More generally, for any integer $k$, one can form the representation up to homotopy $E[-k]$, which is $E$ concentrated in degree $k$.

Example 3.5 (Differential forms). Any closed form $\omega \in \Omega^{n}(A)$ induces a representation up to homotopy on the complex which is the trivial line bundle in degrees 0 and $n$ and zero otherwise. The structure operator is $\nabla^{\text {flat }}+\omega$ where $\nabla^{\text {flat }}$ is the flat connection on the trivial line bundle. If $\omega$ and $\omega^{\prime}$ are cohomologous, then the resulting representations up to homotopy are isomorphic with isomorphism defined by $\Phi_{0}=\mathrm{Id}, \Phi_{n-1}=\theta \in \Omega^{n-1}(A)$ chosen so that $d(\theta)=\omega-\omega^{\prime}$. In conclusion, we have a well defined map $H^{\bullet}(A) \rightarrow \operatorname{Rep}^{\infty}(A)$.

Example 3.6 (Conjugation). For any representation up to homotopy $E$ with structure operator $D$ given by (10), one can form a new representation up to homotopy $\bar{E}$, which has the same underlying graded vector bundle as $E$, but with the structure operator

$$
\bar{D}=-\partial+\nabla-\omega_{2}+\omega_{3}-\omega_{4}+\ldots
$$

In general, $E$ and $\bar{E}$ are isomorphic, with isomorphism $\Phi=\Phi_{0}$ equal to $(-1)^{n} \operatorname{Id}$ on $E^{n}$.

Remark 3.7. It is worth being more explicit on the building blocks of representations up to homotopy which are concentrated in two consecutive degrees, say 0 and 1. From Proposition 3.2 we see that such a representation consists of

1. Two vector bundles $E$ and $F$, and a vector bundle map $f: E \rightarrow F$.

2. $A$-connections on $E$ and $F$, both denoted $\nabla$, compatible with $\partial\left(\nabla_{\alpha} \partial=\partial \nabla_{\alpha}\right)$.

3. A 2-form $K \in \Omega^{2}(A ; \operatorname{Hom}(F, E))$ such that

$$
R_{\nabla^{E}}=\partial \circ K, R_{\nabla^{F}}=K \circ \partial
$$

and such that $d_{\nabla}(K)=0$.

Example 3.8 (The double of a vector bundle). Let $E$ be a vector bundle over $M$. For any $A$-connection $\nabla$ on $E$ with curvature $R_{\nabla} \in \Omega^{2}(A$; End $(E))$, the complex $E \stackrel{\text { Id }}{\rightarrow} E$ concentrated in degrees 0 and 1 , together with the structure operator

$$
D_{\nabla}:=\mathrm{Id}+\partial+R_{\nabla}
$$


defines a representation up to homotopy of $A$ denoted $\mathcal{D}_{E, \nabla}$. The resulting element

$$
\mathcal{D}_{E} \in \operatorname{Rep}^{\infty}(A)
$$

does not depend on the choice of the connection. To see this, remark that if $\nabla^{\prime}$ is another $A$-connection, then there is an isomorphism

$$
\Phi:\left(\mathcal{D}_{E}, D_{\nabla}\right) \rightarrow\left(\mathcal{D}_{E}, D_{\nabla^{\prime}}\right)
$$

with two components:

$$
\Phi^{0}=\mathrm{Id}, \Phi^{1}(\alpha)=\nabla_{\alpha}-\nabla_{\alpha}^{\prime} .
$$

Example 3.9 (The adjoint representation). It is now clear that the properties of the basic connections and the basic curvature given in Proposition 2.10 give the adjoint complex the structure of a representation up to homotopy. Choosing a connection on the vector bundle $A$, the chain complex

$$
A \stackrel{\rho}{\rightarrow} T M
$$

together with the structure operator

$$
D_{\nabla}:=\rho+\nabla^{\text {bas }}+R^{\text {bas }}
$$

becomes a representation up to homotopy of $A$, denoted $A d_{\nabla}$. The isomorphism class of this representation is called the adjoint representation of $A$ and is denoted

$$
\operatorname{Ad} \in \operatorname{Rep}^{\infty}(A) .
$$

Theorem 3.10. Ad $\in \operatorname{Rep}^{\infty}(A)$ does not depend on the choice of the connection. Also, there is a natural isomorphism:

$$
H^{\bullet}(A ; A d) \cong H_{d e f}^{\bullet}(A) .
$$

Proof. Let $\nabla^{\prime}$ be another connection. Then $\Phi=\Phi_{0}+\Phi_{1}$ with

$$
\Phi_{0}=\mathrm{Id}, \Phi_{1}(\alpha) X=\nabla_{X}(\alpha)-\nabla_{X}^{\prime}(\alpha)
$$

defines an isomorphism between the resulting representations. For the last part, note that there is an exact sequence

$$
0 \rightarrow \Omega^{n}(A ; A) \rightarrow C_{\mathrm{def}}^{n}(A) \stackrel{-\sigma}{\rightarrow} \Omega^{n-1}(A ; T M) \rightarrow 0,
$$

where $\sigma$ is the symbol map (see Subsection 2). Moreover, a connection $\nabla$ on $A$ induces a splitting of this sequence, and then an isomorphism

$$
\begin{aligned}
\Psi: C_{\mathrm{def}}^{n}(A) & \rightarrow \Omega^{n}(A ; A) \oplus \Omega^{n-1}(A ; T M)=\Omega(A, \mathrm{Ad})^{n} \\
D & \mapsto\left(c_{D},-\sigma_{D}\right)
\end{aligned}
$$

where $\sigma_{D}$ is the symbol of $D$ and $c_{D}$ is given by

$$
c_{D}\left(\alpha_{1}, \ldots, \alpha_{k}\right)=D\left(\alpha_{1}, \ldots, \alpha_{k}\right)+(-1)^{k-1} \sum_{i=1}^{k}(-1)^{i} \nabla_{\sigma(D)\left(\alpha_{1}, \ldots, \widehat{\alpha_{i}}, \ldots, \alpha_{k}\right)}\left(\alpha_{i}\right) .
$$

This map is an isomorphism of $\Omega(A)$-modules. We need to prove that the operators $\delta$ and $D_{\nabla}$ coincide. Since these two operators are derivations with respect to the module structures, it is enough to prove that they coincide in low degrees and this is a simple check. 
Example 3.11 (Restrictions of the adjoint representation). Given a Lie algebroid $A$ and a leaf $L \subset M$ of $A$, the restriction of the adjoint representation of $A$ defines a representation up to homotopy

$$
\left.\operatorname{Ad}\right|_{L} \in \operatorname{Rep}^{\infty}\left(A_{L}\right) .
$$

The resulting cohomology $H^{\bullet}\left(A_{L} ;\left.\mathrm{Ad}\right|_{L}\right)$ will be denoted by $H_{\text {def, } L}^{*}(A)$ and will be called the restricted deformation cohomology (see below). Note that, unlike the previous example (or most of the coming ones), there does not seem to be any "intrinsic complex" computing this cohomology. It relates to the deformation cohomology in the following way.

Proposition 3.12. There is a long exact sequence:

$$
\cdots \rightarrow H_{d e f}^{n}\left(A_{L}\right) \rightarrow H_{d e f, L}^{n}(A) \stackrel{\phi}{\rightarrow} H^{n-1}\left(A_{L} ; \nu_{L}\right) \rightarrow H_{d e f}^{n+1}(A) \rightarrow \cdots
$$

Proof. Choose a connection $\nabla$ on $A$ and use it to represent $\operatorname{Ad}_{A}$. Use the restriction of $\nabla$ to $L$ to represent the adjoint representation of $A_{L}$. Then, there is a short exact sequence of representations up to homotopy

$$
\left.\operatorname{Ad}_{A_{L}} \rightarrow \operatorname{Ad}_{A}\right|_{L} \rightarrow \nu_{L}[1]
$$

where $\nu_{L}$ is the representation of $A_{L}$ given by the Bott-type connection.

Finally, let us point out here that $H_{\text {def, } L}^{*}(A)$ controls the strongy stability of $L$ as a leaf of $A$. This appears implicitely in [10]. More precisely, using the same techniques as in loc. cit., one can show the following type of result: if $L$ is a compact leaf of a Lie algebroid $A$, satisfying $H_{\mathrm{def}, L}^{2}(A)=0$, then $L$ is strongly stable. More precisely, any Lie algebroid which is close enough to $A$ has a family of leaves parametrized by $H_{\text {def, } L}^{1}(A)$ with the property that they are diffeomorphic to $L$ and the induced transitive Lie algebroids are isomorphic to $\left.A\right|_{L}$. Moreover, the Lie algebroid version of the conjecture of [10] takes the following form: if $L$ is a compact leaf of a Lie algebroid $A$ and the canonical map $\phi: H_{\text {def, } L}^{2}(A) \rightarrow H^{1}\left(A_{L} ; \nu_{L}\right)$ vanishes, then $L$ is stable, i.e. any Lie algebroid which is close enough to $A$ has at least one leaf diffeomorphic to $L$ (this stability is proven in loc. cit. under the weaker assumption that $\left.H^{1}\left(A_{L} ; \nu_{L}\right)=0\right)$.

Example 3.13 (Extensions). We will now explain how representations up to homotopy of length 1 are related to extensions.

Proposition 3.14. For any representation up to homotopy of length one with vector bundles $E$ in degree 0 and $F$ in degree 1 and structure operator $D=\partial+\nabla+K$, there is an extension of Lie algebroids:

$$
\mathfrak{g}_{\partial} \rightarrow \tilde{A} \rightarrow A
$$

where

1. $\mathfrak{g}_{\partial}=\operatorname{Hom}(F, E)$, is a bundle of Lie algebras with bracket $[S, T]_{\partial}=S \partial T-T \partial S$.

2. $\tilde{A}=\mathfrak{g}_{\partial} \oplus A$ with anchor $(S, \alpha) \mapsto \rho(\alpha)$ and bracket

$$
[(S, \alpha),(T, \beta)]=\left([S, T]+\nabla_{\alpha}(T)-\nabla_{\beta}(S)+K(\alpha, \beta),[\alpha, \beta]\right) .
$$

Proof. After a careful computation, we find that the Jacobi identity for the bracket of $\tilde{A}$ brakes into the following equations (cf. Theorem 7.3.7 in [22]):

$$
\begin{gathered}
\nabla_{\alpha}([S, T])=\left[\nabla_{\alpha}(S), T\right]+\left[S, \nabla_{\alpha}(T)\right] \\
\nabla_{[\beta, \gamma]}(T)-\nabla_{\beta} \nabla_{\gamma}(T)+\nabla_{\gamma} \nabla_{\beta}(T)=[T, K(\beta, \gamma)] \\
K([\alpha, \beta], \gamma)+K([\beta, \gamma], \alpha)+K([\gamma, \alpha], \beta)=\nabla_{\beta}(K(\gamma, \alpha))+\nabla_{\alpha}(K(\beta, \gamma))+\nabla_{\gamma}(K(\alpha, \beta))
\end{gathered}
$$

for $\alpha, \beta, \gamma \in \Gamma(A)$ and $S, T \in \Gamma\left(\mathfrak{g}_{\partial}\right)$. These equations are not equivalent to, but they follow from the equations satisfied by $\partial, \nabla$ and $K$. The first equation follows from the compatibility of $\partial$ and $\nabla$, the second equation follows from the two equations satisfied by the curvature, while the last equation is precisely $d_{\nabla}(K)=0$. 
Remark 3.15. One can show that isomorphic length one representations up to homotopy induce isomorphic extensions.

Example 3.16. When $A=T M$ and $E$ is a vector bundle, the extension associated to the double of $E$ (Proposition 3.8) is isomorphic to the "Atiyah extension" induced by $E$ :

$$
\operatorname{End}(E) \rightarrow \mathfrak{g l}(E) \rightarrow T M
$$

This extension is discussed e.g. in Section 1 of [21] (where $\mathfrak{g l}(E)$ is denoted by $\mathcal{D}(E)$ ). Recall that $\mathfrak{g l}(E)$ is the vector bundle over $M$ whose sections are the derivations of $E$, i.e. pairs $(D, X)$ consisting of a linear map $D: \Gamma(E) \rightarrow \Gamma(E)$ and a vector field $X$ on $M$, such that $D(f s)=f D(s)+L_{X}(f) s$ for all $f \in C^{\infty}(M), s \in \Gamma(E)$. The Lie bracket of $\mathfrak{g l}(E)$ is the commutator

$$
\left[(D, X),\left(D^{\prime}, X^{\prime}\right)\right]=\left(D \circ D^{\prime}-D^{\prime} \circ D,\left[X, X^{\prime}\right]\right),
$$

while the anchor sends $(D, X)$ to $X$. A connection on $E$ is the same thing as a splitting of the Atiyah extension, and it induces an identification

$$
\mathfrak{g l}(E) \cong \operatorname{End}(E) \oplus T M .
$$

Computing the bracket (or consulting [21]) we find that, after this identification, the Atiyah extension becomes the extension associated to the double $\mathcal{D}_{E}$.

Example 3.17. The extension associated to the adjoint representation is the one given by the first jet algebroid $J^{1}(A)$. This follows from a computation similar to the previous one, or by using the identities in [4]. In our view, this is the precise relation between the adjoint representation and $J^{1}(A)$. We emphasize here that there doesn't seem to be any construction which associates to a Lie algebroid extension of $A$ a representation up to homotopy so that, applying it to $J^{1}(A)$ one recovers the adjoint representation. In other words, $J^{1}(A)$ with its structure of extension of $A$ does not contain all the information about the structure of the adjoint representation.

Example 3.18 (Representations up to homotopy of $T M$ ). The representations up to homotopy of $T M$ are connections on complexes of vector bundles which are flat up to homotopy. Indeed, at least the first equation in Proposition 3.2 says that the curvature of $\nabla$ is trivial cohomologically (up to homotopy).

On the other hand, a flat connection $\nabla$ on a vector bundle $E$ can be integrated to a representation of the fundamental group(oid) of $M$. This correspondence is induced by parallel transport. To be more precise, given a vector bundle $E$ endowed with a connection $\nabla$, for any path $\gamma$ in $M$ from $x$ to $y$, the parallel transport along $\gamma$ with respect to $\nabla$ induces a linear isomorphism

$$
T_{\gamma}: E_{x} \rightarrow E_{y}
$$

This construction is compatible with path concatenation. When $\nabla$ is flat, $T_{\gamma}$ only depends on the homotopy class of $\gamma$, and this defines an action of the homotopy group(oid) of $M$ on $E$. In is only natural to ask what connections which are flat up homotopy integrate to.

Proposition 3.19. Let $(E, D)$ be a representation up to homotopy of TM. Then:

1. For any path $\gamma$ in $M$ from $x$ to $y$, there is an induced chain map

$$
T_{\gamma}:\left(E_{x}, \partial\right) \rightarrow\left(E_{y}, \partial\right)
$$

and this construction is compatible with path concatenation. More precisely, $T_{\gamma}$ is the parallel transport with respect to the connection underlying $D$.

2. If $\gamma_{0}$ and $\gamma_{1}$ are two homotopic paths in $M$ from $x$ to $y$ then $T_{\gamma_{0}}$ and $T_{\gamma_{1}}$ are chain homotopic. More precisely, for any homotopy $h$ between $\gamma_{0}$ and $\gamma_{1}$ there is an associated map of degree $-1, T_{h}: E_{x} \rightarrow E_{y}$, such that

$$
T_{\gamma_{1}}-T_{\gamma_{0}}=\left[\partial, T_{h}\right]
$$


Proof. The compatibility of $\nabla$ with the grading and $\partial$ implies that the parallel transport $T_{\gamma}$ is a map of chain complexes. We now prove (2). Given a path $u: I \rightarrow E(I=[0,1])$, sitting over some base path $\gamma: I \rightarrow M$, we denote by

$$
\frac{D u}{D t}=\nabla_{\frac{d \gamma}{d t}}(u)
$$

the derivative of $u$ with respect to the connection $\nabla$. Then, for any path $\gamma$, and any $s, t$, the parallel transport

$$
T_{\gamma}^{s, t}: E_{\gamma(s)} \rightarrow E_{\gamma(t)}
$$

is defined by the equation

$$
\frac{D}{D t} T_{\gamma}^{s, t}(u)=0, \quad T_{\gamma}^{s, s}(u)=u .
$$

The global parallel transport along $\gamma, T_{\gamma}: E_{x} \rightarrow E_{y}$ is obtained for $s=0, t=1$. Note that, for a path in the fiber above $\gamma(s), \phi: I \rightarrow E_{\gamma(s)}$, one has

$$
\frac{D}{D t} T_{\gamma}^{s, t}(\phi(t))=T_{\gamma}^{s, t}\left(\frac{d \phi}{d t}(t)\right)
$$

This implies that, for a path $v: I \rightarrow E$ above $\gamma$ and $u_{0} \in E_{x}$, the (unique) solution of the equation

$$
\frac{D u}{d t}=v, u(0)=u_{0}
$$

can be written in terms of the parallel transport as

$$
u(t)=T_{\gamma}^{0, t}\left(u_{0}+\int_{0}^{t} T_{\gamma}^{t^{\prime}, 0}\left(v\left(t^{\prime}\right)\right) d t^{\prime}\right) .
$$

For any map $u: I \times I \rightarrow E$ sitting above some $h: I \times I \rightarrow M$, we have

$$
\frac{D^{2} u}{D t D \epsilon}-\frac{D^{2} u}{D \epsilon D t}=R\left(\frac{d h}{d t}, \frac{d h}{d \epsilon}\right)(u(\epsilon, t))
$$

where $R=R_{\nabla}$ is the curvature of $\nabla$. Let now $h$ be as in the statement, $u_{0} \in E_{x}$, and consider in the previous equation applied to

$$
u(\epsilon, t)=T_{\gamma_{\epsilon}}^{0, t}\left(u_{0}\right)
$$

We find

$$
\frac{D}{D t}\left(\frac{D u}{D \epsilon}\right)=R\left(\frac{d h}{d t}, \frac{d h}{d \epsilon}\right) u,
$$

where $\gamma_{\epsilon}=h(\epsilon, \cdot)$. Since $\frac{D u}{D \epsilon}(\epsilon, 0)=0$, we find

$$
\frac{D u}{D \epsilon}=T_{\gamma_{\epsilon}}^{0, t} \int_{0}^{t} T_{\gamma_{\epsilon}}^{t^{\prime}, 0} R\left(\frac{d h}{d t}, \frac{d h}{d \epsilon}\right) u\left(\epsilon, t^{\prime}\right) d t^{\prime}
$$

Fixing the argument $t$, since

$$
u(0, t)=T_{\gamma_{0}}^{0, t}\left(u_{0}\right)
$$

by the same argument as above, we deduce that

$$
u(\epsilon, t)=T_{h_{t}}^{0, \epsilon}\left[T_{\gamma_{0}}^{0, t}\left(u_{0}\right)+\int_{0}^{\epsilon} T_{h_{t}}^{\epsilon^{\prime}, 0} T_{\gamma_{\epsilon^{\prime}}}^{0, t} \int_{0}^{t} T_{\gamma_{\epsilon^{\prime}}}^{t^{\prime}, 0} R\left(\frac{d h}{d t}, \frac{d h}{d \epsilon}\right) u\left(\epsilon^{\prime}, t^{\prime}\right) d t^{\prime} d \epsilon^{\prime}\right],
$$

where $h_{t}(\cdot)=h(\cdot, t)$. Taking $\epsilon=1, t=1$, we find

$$
T_{\gamma_{1}}\left(u_{0}\right)=T_{\gamma_{0}}\left(u_{0}\right)+\int_{0}^{1} \int_{0}^{1} T_{\gamma_{\epsilon}}^{t, 1} R\left(\frac{d h}{d t}, \frac{d h}{d \epsilon}\right) T_{\gamma_{\epsilon}}^{0, t}\left(u_{0}\right) d t d \epsilon .
$$


Using now that $R+\partial\left(\omega_{2}\right)=0$, we deduce that

$$
T_{\gamma_{1}}\left(u_{0}\right)-T_{\gamma_{0}}\left(u_{0}\right)=\left[\partial, T_{h}\right] u_{0},
$$

where $T_{h} \in \operatorname{Hom}\left(E_{x}, E_{y}\right)$ is

$$
T_{h}=-\int_{0}^{1} \int_{0}^{1} T_{\gamma_{\epsilon}}^{t, 1} \omega_{2}\left(\frac{d h}{d t}, \frac{d h}{d \epsilon}\right) T_{\gamma_{\epsilon}}^{0, t} d t d \epsilon
$$

Note that the expression under the integral is in the $(\epsilon, t)$-independent vector space $\operatorname{Hom}\left(E_{x}, E_{y}\right)$.

Remark 3.20. If we want to pass to the homotopy groupoid then we choose a representative $\gamma_{a}$ for every element $a$ in the fundamental groupoid of $M$, choose a homotopy $h_{a, b}$ between $\gamma_{a b}$ and $\gamma_{a} * \gamma_{b}$ for any two $a$ and $b$ (etc). Define the action of $a$ on $E$ by

$$
a \cdot v=T_{\gamma_{a}}(v) .
$$

This is not a true action because the associativity is not respected. However, the previous proposition implies that

$$
a \cdot(b \cdot v)-(a b) \cdot v=[\partial, T(a, b)] u
$$

where $T(a, b)=T_{h_{a, b}}$. This is just the first equation in an infinite list of equations. Although the precise notion of representations up to homotopy of groupoids has not been introduced yet (it will be discussed in [2]), this shows, at least intuitively, that a connection which is flat up to homotopy integrates to "a representation up to homotopy of the fundamental groupoid of $M "$.

\subsection{Operations and more examples}

We have already seen in subsections 2.3 and 2.4 that the standard operations with vector spaces such as

$$
\begin{gathered}
E \mapsto E^{*}, E \mapsto \Lambda(E), E \mapsto S(E), \\
(E, F) \mapsto E \oplus F,(E, F) \mapsto E \otimes F,(E, F) \mapsto \underline{\operatorname{Hom}}(E, F)
\end{gathered}
$$

extend to the setting of graded vector bundles, complexes of vector bundles and complexes of vector bundles endowed with a connection. The extension to representations up to homotopy is now straightforward.

Example 3.21 (Taking duals). For $E \in \mathbb{R e p}^{\infty}(A)$ with associated structure operator $D$, the operator $D^{*}$ corresponding to the dual $E^{*}$ is uniquely determined by the condition

$$
d_{A}\left(\eta \wedge \eta^{\prime}\right)=D^{*}(\eta) \wedge \eta^{\prime}+(-1)^{|\eta|} \eta \wedge D\left(\eta^{\prime}\right),
$$

for all $\eta \in \Omega\left(A ; E^{*}\right)$ and $\eta^{\prime} \in \Omega(A ; E)$, where $\wedge$ is the operation

$$
\Omega\left(A ; E^{*}\right) \otimes \Omega(A ; E) \rightarrow \Omega(A)
$$

induced by the pairing between $E^{*}$ and $E$ (see subsection 2.3). In terms of the components of $D$, if $D=\partial+\nabla+\sum_{i \geq 2} \omega_{i}$, we find $D^{*}=\partial^{*}+\nabla^{*}+\sum_{i \geq 2} \omega_{i}^{*}$, where $\nabla^{*}$ is the connection dual to $\nabla$ and, for $\eta_{k} \in\left(E^{k}\right)^{*}$,

$$
\partial^{*}(\eta)=-(-1)^{k} \eta \circ \partial, \omega_{p}^{*}\left(\alpha_{1}, \ldots, \alpha_{p}\right)\left(\eta_{k}\right)=-(-1)^{k(p+1)} \eta_{k} \circ \omega_{p}\left(\alpha_{1}, \ldots, \alpha_{p}\right) .
$$

In particular, when starting with a representation up to homotopy of length one, $D=$ $\partial+\nabla+K$ on $E \stackrel{\partial}{\rightarrow} F(E$ in degree 0 and $F$ in degree 1$)$, the dual complex will be $F^{*} \stackrel{\partial^{*}}{\rightarrow} E^{*}$ $\left(F^{*}\right.$ in degree -1 and $E^{*}$ in degree 0$)$, with $D^{*}=\partial^{*}+\nabla^{*}-K^{*}$. The fact that some signs appear when taking duals is to be expected since, for any connection $\nabla$, the curvature of $\nabla^{*}$ equals the negative of the dual of the curvature of $\nabla$. 
Example 3.22 (Tensor products). For $E, F \in \mathbb{R e p}^{\infty}(A)$, with associated structure operators $D^{E}$ and $D^{F}$, the operator $D$ corresponding to $E \otimes F$ is uniquely determined by the condition

$$
D\left(\eta_{1} \wedge \eta_{2}\right)=D^{E}\left(\eta_{1}\right) \wedge \eta_{2}+(-1)^{\left|\eta_{1}\right|} \eta_{1} \wedge D^{F}\left(\eta_{2}\right),
$$

for all $\eta_{1} \in \Omega(A ; E)$ and $\eta_{2} \in \Omega(A ; F)$. More explicitly, if $D^{E}=\partial^{E}+\nabla^{E}+\omega_{2}^{E}+\cdots$ and similarly for $D^{F}$, then $D=\partial+\nabla+\omega_{2}+\cdots$, where:

1. $\partial$ is just the tensor product of $\partial^{E}$ and $\partial^{F}: \partial=\partial^{E} \otimes \operatorname{Id}+\operatorname{Id} \otimes \partial^{F}$,

$$
\partial(u \otimes v)=\partial^{E}(u) \otimes v+(-1)^{|u|} u \otimes \partial^{F}(v)
$$

2. $\nabla$ is just the tensor product connection of $\nabla^{E}$ and $\nabla^{F}: d_{\nabla}=d_{\nabla^{E}} \otimes \operatorname{Id}+\operatorname{Id} \otimes d_{\nabla^{F}}$,

$$
\nabla_{\alpha}(u \otimes v)=\nabla_{\alpha}^{E}(u) \otimes v+u \otimes \nabla_{\alpha}^{F}(v) .
$$

3. $\omega_{p}=\omega_{p}^{E} \otimes \operatorname{Id}+\operatorname{Id} \otimes \omega_{p}^{F}$.

Example 3.23 (Pull-back). A Lie algebroid $A$ over $M$ can be pulled-back along submersion $\tau: N \rightarrow M$ or, more generally, along smooth maps $\tau$ which satisfy certain condition (see below). Recall [23] that the pull-back algebroid $\tau^{!} A$ has the fiber at $x \in N$ :

$$
\tau^{!}(A)_{x}=\left\{(X, \alpha): X \in T_{x} N, \alpha \in A_{\tau(x)},(d \tau)(X)=\rho(\alpha)\right\} .
$$

The condition mentioned above is that this is a smooth vector bundle over $N$, which certainly happens if $\tau$ is a submersion or the inclusion of an orbit of $A$. The anchor of $\tau^{!} A$ sends $(X, \alpha)$ to $X$, while the bracket is uniquely determined by the derivation rule and

$$
\left[\left(X, \tau^{*} \alpha\right),\left(Y, \tau^{*} \beta\right)\right]=\left([X, Y], \tau^{*}[\alpha, \beta]\right) .
$$

In general, there is a pull-back map (functor)

$$
\tau^{*}: \mathbb{R e p}^{\infty}(A) \rightarrow \mathbb{R e p}^{\infty}\left(\tau^{!}(A)\right)
$$

which sends $E$ with structure operator $D=\partial+\nabla+\sum \omega_{i}$ to $\tau^{*}(E)$ endowed with $D=$ $\partial+\tau^{*}(\nabla)+\sum \tau^{*}\left(\omega_{i}\right)$ where $\tau^{*} \nabla$ is the pull-back connection

$$
\left(\tau^{*} \nabla\right)_{(X, \alpha)}\left(\tau^{*}(s)\right)=\tau^{*}\left(\nabla_{\alpha}(s)\right)
$$

while

$$
\tau^{*}\left(\omega_{i}\right)\left(\left(X_{1}, \alpha_{1}\right), \ldots,\left(X_{i}, \alpha_{i}\right)\right)=\omega_{i}\left(\alpha_{1}, \ldots, \alpha_{i}\right) .
$$

Remark 3.24 (DG algebra picture). The notion of representation up to homotopy can be recasted in the language of differential graded modules over differential graded algebras [17], and some of the constructions in this paper may be seen as particular cases of the general theory. We emphasize however that we insist on working with DG-modules which are sections of vector bundles.

Example 3.25 (Semidirect products with representations up to homotopy). If $\mathfrak{g}$ is a Lie algebra and $V \in \mathbb{R e p}{ }^{\infty}(\mathfrak{g})$, the operator making $\Lambda\left(V^{*}\right)$ a representation up to homotopy of $\mathfrak{g}$ is a derivation on the algebra

$$
\Lambda\left(\mathfrak{g}^{*}\right) \otimes \Lambda\left(V^{*}\right)=\Lambda\left((\mathfrak{g} \oplus V)^{*}\right),
$$

i.e. defines the structure of $L_{\infty}$-algebra (see [27]) on the direct sum $\mathfrak{g} \oplus V$. This $L_{\infty}$-algebra deserves the name "semi-direct product of $\mathfrak{g}$ and $V$ ", and is denoted $\mathfrak{g} \ltimes V$ (it is the usual semi-direct product if $V$ is just a usual representation). 
Example 3.26 (Exterior powers of Ad and $k$-differentials). Applying the exterior powers construction to the adjoint representation, we find new elements:

$$
\Lambda^{k} \operatorname{Ad} \in \operatorname{Rep}^{\infty}(A),
$$

one for each positive integer $k$. These are given by the representations up to homotopy $\Lambda^{k}\left(\operatorname{Ad}_{\nabla}\right)$, where $\nabla$ is an arbitrary connection on $A$. Generalizing the case of the cohomology of $A$ with coefficients in the adjoint representation Ad, we now show that the cohomology with coefficients in $\Lambda^{k} \mathrm{Ad}$ can be computed by an intrinsic complex (which does not require the use of a connection). More precisely, we define $\left(C^{\bullet}\left(A ; \Lambda^{k} \mathrm{Ad}\right), d\right)$ as follows. An element $c \in C^{p}\left(A ; \Lambda^{k} \mathrm{Ad}\right)$ is a string $c=\left(c_{0}, c_{1}, \ldots\right)$ where

$$
c_{i}: \underbrace{\Gamma(A) \times \ldots \times \Gamma(A)}_{(p-i) \text { times }} \rightarrow \Gamma\left(\Lambda^{k-i} A \otimes S^{i} T M\right),
$$

are multilinear, antisymmetric maps related by:

$$
\left.c_{i}\left(\alpha_{1}, \ldots, f \alpha_{p-i}\right)=f c_{i}\left(\alpha_{1}, \ldots, f \alpha_{p-i}\right)+i(d f)\left(c_{i+1}\left(\alpha_{1}, \ldots, \alpha_{p-i-1}\right) \wedge \alpha_{p-i}\right)\right)
$$

where $i(d f): S^{i+1}(T M) \rightarrow S^{i}(T M)$ is the contraction by $d f$. We think of $c_{1}, c_{2}, \ldots$ as the tail of $c_{0}$, which measures the failure of $c_{0}$ to be $C^{\infty}(M)$-linear. For instance, to define the differential $d c$, we first define its leading term by the Koszul formula

$$
\begin{aligned}
(d c)_{0}\left(\alpha_{1}, \ldots, \alpha_{p+1}\right) & =\sum_{i<j}(-1)^{i+j} c_{0}\left(\left[\alpha_{i}, \alpha_{j}\right], \ldots, \hat{\alpha_{i}}, \ldots, \hat{\alpha_{j}}, \ldots, \alpha_{p+1}\right)+ \\
& +\sum_{i}(-1)^{i+1} L_{\rho\left(\alpha_{i}\right)}\left(c_{0}\left(\alpha_{1}, \ldots, \hat{\alpha_{i}}, \ldots, \alpha_{p+1}\right)\right)
\end{aligned}
$$

and then the tail can be computed by applying the principle we have mentioned above. The following proposition provides a cohomological framework for the notion of a $k$-differential (see Remark 2.7).

Proposition 3.27. The cohomology $H^{\bullet}\left(A ; \Lambda^{k} A d\right)$ is naturally isomorphic to the cohomology of the complex $\left(C^{\bullet}\left(A ; \Lambda^{k} A d\right), d\right)$. Moreover, the 1-cocycles of this complex are precisely the $k$-differentials on $A$.

Proof. For the first part, we pick a connection $\nabla$ to realize the adjoint representation and we claim that the complexes $\left(C^{\bullet}\left(A ; \Lambda^{k} \mathrm{Ad}\right), d\right)$ and $\left(\Omega^{\bullet}\left(A ; \Lambda^{k} \mathrm{Ad}\right), D_{\nabla}\right)$ are isomorphic. For $k=0$ the statement is trivial while the case $k=1$ follows from Theorem 3.10. The general statement follows from these two cases if one observes that, both $\oplus_{k} C^{\bullet}\left(A ; \Lambda^{k} \mathrm{Ad}\right)$ and $\oplus_{k} \Omega^{\bullet}\left(A ; \Lambda^{k} \mathrm{Ad}\right)$ are algebras generated in low degree for which the corresponding differentials are derivations. For the second part, remark that an element in $C^{1}\left(A ; \Lambda^{k} \mathrm{Ad}\right)$ is a pair $\left(c_{0}, c_{1}\right)$ where $c_{0}: \Gamma(A) \rightarrow \Gamma\left(\Lambda^{k} A\right)$ and $c_{1} \in \Gamma\left(\Lambda^{k-1} A \otimes T M\right)$ satisfy the appropriate equation. Viewing $c_{1}$ as the map $C^{\infty}(M) \rightarrow \Gamma\left(\Lambda^{k-1} A\right), f \mapsto i(d f)\left(c_{1}\right)$, we see that the elements of $C^{1}\left(A ; \Lambda^{k} \mathrm{Ad}\right)$ are precisely the almost $k$-differentials on $A$. The fact that the cocycle equation is precisely the $k$-differential equation follows by a simple computation.

Example 3.28 (The coadjoint representation). The dual of the adjoint representation of a Lie algebroid $A$ is called the coadjoint representation of $A$, denoted $\mathrm{Ad}^{*}$. Using a connection $\nabla$ on $A$, it is given by the representation up to homotopy

$$
\mathrm{Ad}^{*}: \underbrace{T^{*} M}_{\text {degree }-1} \stackrel{\rho^{*}}{\rightarrow} \underbrace{A^{*}}_{\text {degree } 0}, \quad D=\rho^{*}+\left(\nabla^{\text {bas }}\right)^{*}-\left(R_{\nabla}^{\text {bas }}\right)^{*} .
$$

As in the case of the adjoint representation, the resulting cohomology can be computed by an "intrinsic complex" (which does not require the choice of a connection). This complex, 
denoted $C^{\bullet}\left(A ; \mathrm{Ad}^{*}\right)$, is defined as follows. An element in $C^{p}\left(A ; \mathrm{Ad}^{*}\right)$ is a pair $c=\left(c_{0}, c_{1}\right)$ where $c_{0}$ is a multilinear antisymmetric map

$$
c_{0}: \underbrace{\Gamma(A) \times \ldots \times \Gamma(A)}_{p \text { times }} \rightarrow \Omega^{1}(M),
$$

and $c_{1} \in \Omega^{p-1}\left(A ; A^{*}\right)$ is such that

$$
c_{0}\left(\alpha_{1}, \ldots, \alpha_{p-1}, f \alpha_{p}\right)=f c_{0}\left(\alpha_{1}, \ldots, \alpha_{p-1}, \alpha_{p}\right)-d f \wedge c_{1}\left(\alpha_{1}, \ldots, \alpha_{p-1}\right)\left(\alpha_{p}\right),
$$

for all $f \in C^{\infty}(M), \alpha_{i} \in \Gamma(A)$. The differential of $c, d(c) \in C^{p+1}\left(A ; \mathrm{Ad}^{*}\right)$ is given by the Koszul-type formulas

$$
\begin{aligned}
(d c)_{0}\left(\alpha_{1}, \ldots, \alpha_{p+1}\right) & =\sum_{i<j}(-1)^{i+j} c_{0}\left(\left[\alpha_{i}, \alpha_{j}\right], \ldots, \hat{\alpha_{i}}, \ldots, \hat{\alpha_{j}}, \ldots, \alpha_{p+1}\right)+ \\
& +\sum_{i}(-1)^{i+1} L_{\rho\left(\alpha_{i}\right)}\left(c_{0}\left(\alpha_{1}, \ldots, \hat{\alpha_{i}}, \ldots, \alpha_{p+1}\right)\right), \\
(d c)_{1}\left(\alpha_{1}, \ldots, \alpha_{p}\right)= & \sum_{i<j}(-1)^{i+j} c_{0}\left(\left[\alpha_{i}, \alpha_{j}\right], \ldots, \hat{\alpha_{i}}, \ldots, \hat{\alpha_{j}}, \ldots, \alpha_{p}\right)+ \\
& +\sum_{i}(-1)^{i+1} L_{\rho\left(\alpha_{i}\right)}\left(c_{0}\left(\alpha_{1}, \ldots, \hat{\alpha_{i}}, \ldots, \alpha_{p}\right)\right)+(-1)^{p+1} c\left(\alpha_{1}, \ldots, \alpha_{p}\right) \circ \rho .
\end{aligned}
$$

Proposition 3.29. $\left(C^{\bullet}\left(A ; A d^{*}\right), d\right)$ is a cochain complex whose cohomology is canonically isomorphic to $H^{\bullet}\left(A ; A d^{*}\right)$.

Example 3.30. More generally, for any $q$, the representation up to homotopy $S^{q}\left(\operatorname{Ad}^{*}\right)$ and its cohomology can be treated similarly. This will be made more explicit in our discussion on the Weil algebra.

\subsection{Cohomology, the derived category, and some more examples}

As we already mentioned several times (e.g. in Example 2.6), one of the reasons we work with complexes is that we want to avoid non-smooth vector bundles. The basic idea was that a complex represents its cohomology bundle (typically a graded non-smooth vector bundle). To complete this idea, we need to allow ourselves more freedom when comparing two complexes so that, morally, if they have the same cohomology bundles, then they become equivalent. This will happen in the derived category. For a more general discussion on the derived category of a $D G$ algebra we refer the reader to [26].

Definition 3.31. A morphism $\Phi$ between two representations up to homotopy $E$ and $F$ is called a quasi-isomorphism if the first component of $\Phi$, the map of complexes $\Phi_{0}:(E, \partial) \rightarrow$ $(F, \partial)$, is a quasi-isomorphism. We denote by $\mathbb{D} e r(A)$ the category obtained from $\mathbb{R} e p^{\infty}(A)$ by formally inverting the quasi-isomorphisms, and by Der $(A)$ the set of isomorphisms classes of objects of $\mathbb{D}$ er $(A)$.

Remark 3.32 (Hom in the derived category). Since we work with vector bundles, there is the following simple realization of the derived category. Given two representations up to homotopy $E$ and $F$ of $A$, there is a notion of homotopy between maps from $E$ to $F$. To describe it, we remark that morphisms in $\mathbb{R e p}^{\infty}(A)$ from $E$ to $F$ correspond to 0-cycles in the complex with coefficients in the induced representation up to homotopy $\underline{\operatorname{Hom}}(E, F)$ :

$$
\operatorname{Hom}_{\mathbb{R e p}^{\infty}(A)}(E, F)=Z^{0}(\Omega(A ; \underline{\operatorname{Hom}}(E, F))) .
$$

Two maps $\Phi, \Psi: E \rightarrow F$ in $\mathbb{R e p}^{\infty}(A)$ are called homotopic if there exists a degree -1 map $H: \Omega(A ; E) \rightarrow \Omega(A ; F)$ which is $\Omega(A)$-linear and satisfies $D_{E} H+H D_{F}=\Phi-\Psi$, where $D_{E}$ 
and $D_{F}$ are the structure operators of $E$ and $F$, respectively. We denote by $[E, F]$ the set of homotopy classes of such maps. Hence,

$$
[E, F]:=H^{0}(\Omega(A ; \underline{\operatorname{Hom}}(E, F))) .
$$

As in the case of complexes of vector bundles (see part (2) of Lemma 2.17), and by the same type of arguments, we see that a map $\Phi: E \rightarrow F$ is a quasi-isomorphism if and only if it is a homotopy equivalence. From this, we deduce the following realization of $\operatorname{Der}(A)$ : its objects are the representations up to homotopy of $A$, while

$$
\operatorname{Hom}_{\mathbb{D e r}(A)}(E, F)=[E, F] .
$$

Note that, in this language, for any $F \in \mathbb{R e p}^{\infty}(A)$,

$$
H^{n}(F)=[\mathbb{R}[n], F] .
$$

Also, the mapping cone construction gives a function

$$
\text { Map : }[E, F] \rightarrow \operatorname{Rep}^{\infty}(A)
$$

which, when applied to $E=\mathbb{R}[n]$, gives the construction from Example 3.5.

Example 3.33. If $A=\mathcal{F} \subset T M$ is a foliation on $M$, then the projection from the complex $\mathcal{F} \hookrightarrow T M$ underlying the adjoint representation into $\nu[-1]$ (the normal bundle $\nu=T M / \mathcal{F}$ concentrated in degree 1 ) is clearly a quasi-isomorphism of complexes. It is easy to see that this projection is actually a morphism of representations up to homotopy, when $\nu$ is endowed with the Bott connection (see Example 2.6). Hence, as expected,

$$
\operatorname{Ad}_{\mathcal{F}} \cong \nu[1] \quad(\text { in } \operatorname{Der}(\mathcal{F})) .
$$

Similarly, for a transitive Lie algebroid $A$, i.e. one for which the anchor is surjective,

$$
\operatorname{Ad}_{A} \cong \mathfrak{g}(A) \quad(\text { in } \operatorname{Der}(A))
$$

Next, we will show that the cohomology $H^{\bullet}(A ;-)$, viewed as a functor from the category of representations up to homotopy, descends to the derived category.

Proposition 3.34. Any quasi-isomorphism $\Phi: E \rightarrow F$ between two representations up to homotopy of $A$ induces an isomorphism in cohomology $\Phi: H(A ; E) \rightarrow H(A ; F)$.

Proof. If $E$ is a representation up to homotopy of $A$, one can form a decreasing filtration on $\Omega(A ; E)$ induced by the form-degree

$$
\cdots \subset F^{2}(\Omega(A ; E)) \subset F^{1}(\Omega(A, E)) \subset F^{0}(\Omega(A ; E))=\Omega(A ; E)
$$

where

$$
F^{p}(\Omega(A ; E))=\Omega^{p}(A ; E) \oplus \Omega^{p+1}(A ; E) \oplus \cdots
$$

This filtration induces a spectral sequence $\mathcal{E}$ with

$$
\mathcal{E}_{0}^{p, q}=\Omega^{p}\left(A ; E^{q}\right) \Rightarrow H^{p+q}(A ; E),
$$

where the differential $d_{0}^{p, q}: \mathcal{E}_{0}^{p q} \rightarrow \mathcal{E}_{0}^{p, q+1}$ is induced by the differential $\partial$ of $E$. Given a morphism $\Phi: E \rightarrow F$, one has an induced map of spectral sequences which, at the first level is induced by $\Phi^{0}$. Hence, the assumption that $\Phi$ is a quasi-isomorphism implies that the map induced at the level of spectral sequences is an isomorphism at the second level. We deduce that $\Phi$ induces an isomorphism in cohomology. 
Example 3.35. Applying this result to the first quasi-isomorphism discussed in Example 3.33, we find that the deformation cohomology of a foliation $\mathcal{F}$ is isomorphic to the shifted cohomology of $\mathcal{F}$ with coefficients in $\nu$ - and this is Proposition 4 in [12].

Example 3.36 (Acyclic complexes). As a corollary of the previous proposition, we find that $H^{\bullet}(A ; E)=0$ for any $E \in \operatorname{Rep}^{\infty}(A)$ whose underlying complex $(E, \partial)$ is acyclic. However, one can say much more in this case, exact complexes can naturally be viewed as representations up to homotopy, and they are precisely the representations up to homotopy which are quasi-isomorphic to the trivial (zero) representation. Of course, the double $\mathcal{D}_{E}$ of a vector bundle (Example 3.8) belongs to this class.

Proposition 3.37. Any exact complex $(E, \partial)$ induces a well-defined element in $\operatorname{Rep}^{\infty}(A)$. More precisely, given $(E, \partial)$,

1. There exist $A$-connections $\nabla$ on $(E, \partial)$.

2. For any A-connection $\nabla$ on the complex $E$, one can find $\omega_{[i]}$ for $i \geq 2$ such that

$$
D=\partial+d_{\nabla}+\sum_{i} \omega_{[i]}
$$

gives $E$ the structure of a representation up to homotopy of $A$.

3. Any two representations up to homotopy obtained in this way are isomorphic.

Proof. Choosing a metric on the complex as in Lemma 2.17 we obtain a decomposition $E^{k}=\operatorname{Im}(\partial)^{k} \oplus \operatorname{Im}\left(\partial^{*}\right)^{k}$. Now we choose arbitrary $A$-connections on all the subbundles $\operatorname{Im}\left(\partial^{*}\right)^{k}$ and use the isomorphisms $\partial: \operatorname{Im}\left(\partial^{*}\right)^{k-1} \rightarrow \operatorname{Im}(\partial)^{k}$ to extend them to $E^{k}$. It is clear from the construction that such connections are compatible with $\partial$.

For the existence of $D$, we have to solve the equations

$$
\partial\left(\omega_{k}\right)+d_{\nabla}\left(\omega_{k-1}\right)+\omega_{2} \circ \omega_{k-2}+\omega_{3} \circ \omega_{k-3}+\ldots+\omega_{k-2} \circ \omega_{2}=0 .
$$

This can be done by an inductive cohomological argument, but we can be more explicit here if we choose a contracting homotopy $h$ of $(E, \partial)$ (cf. Lemma 2.17):

$$
h \partial+\partial h=-I d .
$$

It is not difficult to check that

$$
\omega_{n}=h \circ d_{\nabla}(h)^{n-2} \circ R_{\nabla}
$$

satisfy the desired equations. Assume now that $D$ and $D^{\prime}$ define two structures of representations up to homotopy. To distinguish them, we denote $E=(E, D)$ and $E^{\prime}=\left(E, D^{\prime}\right)$. We consider the resulting representation up to homotopy

$$
\mathcal{E}:=\operatorname{Hom}\left(E, E^{\prime}\right)
$$

with associated operator $D^{\mathcal{E}}$. Due to the exactness assumption, $\mathcal{E}$ will be exact. Using the associated filtration (see the proof of Proposition 3.34), we see that $F^{1} \Omega(A ; \mathcal{E})$ must be exact. On the other hand, since $D$ and $D^{\prime}$ have the same underlying complex, we have

$$
D-D^{\prime} \in F^{1} \Omega(A ; \mathcal{E}) .
$$

A simple computation shows that this is actually a cocycle:

$$
D^{\mathcal{E}}\left(D-D^{\prime}\right)=D^{\prime}\left(D-D^{\prime}\right)+\left(D-D^{\prime}\right) D=D^{\prime} D+D^{\prime 2}-D^{2}-D^{\prime} D=0,
$$

hence we find $T \in F^{1}(\Omega(A, \mathcal{E}))$ such that $D^{\mathcal{E}}(T)=D-D^{\prime}$ or, equivalently, $(I d+T) D=$ $D^{\prime}(I d+T)$. Since $T$ is in $F^{1}$, it is nilpotent and therefore $I d+T$ is the desired isomorphism. 
Next, we look at the case where $E$ is a regular representation up to homotopy of $A$ in the sense that the underlying complex $(E, \partial)$ is regular. In this case, the cohomology $\mathcal{H}^{\bullet}(E)$ is a graded vector bundle over $M$ (see subsection 2.4).

Theorem 3.38. Let $E$ be a regular representation up to homotopy of A. Then the equation

$$
\bar{\nabla}_{\alpha}([S]):=\left[\nabla_{\alpha}(S)\right]
$$

for $\alpha \in \Gamma(A)$ and $[S] \in \mathcal{H}^{\bullet}(E)$ makes $\mathcal{H}^{\bullet}(E)$ a representation of $A$. Moreover, the complex $\left(\mathcal{H}^{\bullet}(E), 0\right)$ with connection $\bar{\nabla}$ can be given the structure of a representation up to homotopy of $A$ which is quasi-isomorphic to $E$.

Also, there is a spectral sequence

$$
\mathcal{E}_{2}^{p q}=H^{p}\left(A ; \mathcal{H}^{q}(E)\right) \Rightarrow H^{p+q}(A, E) .
$$

Proof. That $\bar{\nabla}$ is flat follows from the fact that the curvature of $\nabla$ is exact. Also, the spectral sequence is just the one appearing in the previous proof. Next, we have to construct the structure of representation up to homotopy on $\mathcal{H}^{\bullet}(E)$. We will use the notations from the proof of Lemma 2.17 (part 3). The linear Hodge decomposition $E=k e r \Delta+i m(b)+i m\left(b^{*}\right)$ provides quasi-isomorphisms $p: E \rightarrow \mathcal{H}(E)$ and $i: \mathcal{H}(E) \rightarrow E$. The restriction of the Laplacian to $i m(\partial) \oplus i m\left(\partial^{*}\right)$, denoted $\diamond$, is an isomorphism. Then

$$
h=-\diamond^{-1} \partial^{*}
$$

satisfies the following equations:

1. $p \partial=0$,

2. $\partial i=0$,

3. $i p=\mathrm{Id}+h \partial+\partial h$,

4. $h^{2}=0$,

5. $p h=0$.

We denote by the same letters the maps induced at the level of forms: $\partial$ goes from $\Omega(A ; E)$ to $\Omega(A ; E)$, etc, where we use the standard sign conventions. Note that these maps still satisfy the previous equations. We consider

$$
\delta:=D-\partial: \Omega(A ; E) \rightarrow \Omega(A ; E),
$$

where $D$ is the structure operator of $A$. With these,

$$
D_{\mathcal{H}}:=p\left(1+(\delta h)+(\delta h)^{2}+(\delta h)^{3}+\cdots\right) \delta i: \Omega(A ; \mathcal{H}) \rightarrow \Omega(A ; \mathcal{H})
$$

is a degree one operator which squares to zero, and

$$
\Phi:=p\left(1+\delta h+(\delta h)^{2}+(\delta h)^{3}+\cdots\right):(\Omega(A ; E), D) \rightarrow\left(\Omega(A ; \mathcal{H}), D_{\mathcal{H}}\right)
$$

is a cochain map. The assertions about $D_{\mathcal{H}}$ and $\Phi$ follow by direct computation, or by applying the homological perturbation lemma (see e.g. [9]), which also explains our choices. Finally, one remarks that the equations $h^{2}=0$ and $p h=0$ imply that $\Phi$ is $\Omega(A)$-linear and that $D_{\mathcal{H}}$ is a derivation.

Example 3.39 (Serre representations). Any extension of Lie algebras

$$
\mathfrak{l} \rightarrow \tilde{\mathfrak{g}} \rightarrow \mathfrak{g}
$$

induces a representation up to homotopy of $\mathfrak{g}$ with underlying complex the ChevalleyEilenberg complex $\left(C^{\bullet}(\mathfrak{l}), d_{\mathfrak{l}}\right)$ of $\mathfrak{l}$. To describe this representation, we use a splitting $\sigma: \mathfrak{g} \rightarrow \tilde{\mathfrak{g}}$ of the sequence. This induces 
1. For $u \in \mathfrak{g}$, a degree zero operator

$$
\nabla_{u}^{\sigma}:=a d_{\sigma(u)}^{*}: C^{\bullet}(\mathfrak{l}) \rightarrow C^{\bullet}(\mathfrak{l}),
$$

hence a $\mathfrak{g}$-connection $\nabla^{\sigma}$ on $C^{\bullet}(\mathfrak{l})$.

2. The curvature of $\sigma, R_{\sigma} \in C^{2}(\mathfrak{g} ; \mathfrak{l})$ given by

$$
R^{\sigma}(u, v)=[\sigma(u), \sigma(v)]-\sigma([u, v]) .
$$

To produce an $\underline{\text { End }}^{-1}\left(C^{\bullet}(\mathfrak{l})\right)$-valued cochain, we use the contraction operator $i: \mathfrak{l} \rightarrow$ End $^{-1}(C \cdot(\mathfrak{l}))$ (but mind the sign conventions!).

It is now straightforward to check that

$$
D:=d_{\mathfrak{l}}+\nabla^{\sigma}+i\left(R^{\sigma}\right)
$$

makes $C^{\bullet}(\mathfrak{l})$ a representation up to homotopy of $\mathfrak{g}$, with associated cohomology complex isomorphic to $C^{\bullet}(\tilde{\mathfrak{g}})$.

Note that Theorem 3.38 implies that the cohomology of $\mathfrak{l}$ is naturally a representation of $\mathfrak{g}$, and there is spectral sequence with

$$
\mathcal{E}_{2}^{p, q}=H^{p}\left(\mathfrak{g} ; H^{q}(\mathfrak{l})\right) \Longrightarrow H^{p+q}(\tilde{\mathfrak{g}}) .
$$

This is precisely the content of the Serre spectral sequence (see e.g. [28]).

The same argument applies to any extension of Lie algebroids, giving us the spectral sequence of Theorem 7.4.6 in [22].

In the case of representations of length 1 , we obtain the following.

Corollary 3.40. If $(E, D)$ is a representation up to homotopy of $A$ with underlying regular complex $E=E^{0} \oplus E^{1}$ then there is a long exact sequence

$$
\cdots \rightarrow H^{n}\left(A ; \mathcal{H}^{0}(E)\right) \rightarrow H^{n}(A ; E) \rightarrow H^{n-1}\left(A ; \mathcal{H}^{1}(E)\right) \rightarrow H^{n+1}\left(A ; \mathcal{H}^{0}(E)\right) \rightarrow \cdots
$$

Proof. The map

$$
H^{n-1}\left(A ; \mathcal{H}^{1}(E)\right) \rightarrow H^{n+1}\left(A ; \mathcal{H}^{0}(E)\right)
$$

is $d_{2}^{p q}: E_{2}^{p, 1} \rightarrow E_{2}^{p+2,0}$ in the spectral sequence and is given by the wedge product with $\omega_{2}$. The map

$$
H^{n}\left(A ; \mathcal{H}^{0}(E)\right) \rightarrow H^{n}(A ; E)
$$

is determined by the natural inclusion at the level of cochains.

Finally,

$$
H^{n}(A ; E) \rightarrow H^{n-1}\left(A ; \mathcal{H}^{1}(E)\right)
$$

is given at the level of complexes by:

$$
\left(\omega_{0}+\omega_{1}\right) \mapsto \overline{\omega_{1}}
$$

where $\omega_{0} \in \Omega^{n}\left(A ; E^{0}\right)$ and $\omega_{1} \in \Omega^{n-1}\left(A, E^{1}\right)$. Since the spectral sequence collapses at the third stage, we conclude that the sequence is exact.

Example 3.41. Applying the previous corollary to the adjoint representation of a regular Lie algebroid, we obtain the long exact sequence which is Theorem 3 in [12].

\section{The Weil algebra and the BRST model for equivariant cohomology}

In this section we will make use of representations up to homotopy to introduce the Weil algebra associated to a Lie algebroid $A$, generalizing the Weil algebra of a Lie algebra and Kalkman's BRST algebra for equivariant cohomology. We will discuss two versions of the Weil algebra, one which uses a connection and one which is intrinsic. 


\subsection{The connection dependent model}

This subsection is devoted to the explicit model which makes use of a connection. Let $\nabla$ be a connection on the vector bundle $A$.

Define the algebra

$$
W(A, \nabla)=\bigoplus_{u, v, w} \Gamma\left(\Lambda^{u} T^{*} M \otimes S^{v}\left(A^{*}\right) \otimes \Lambda^{w}\left(A^{*}\right)\right) .
$$

It is graded by the total degree $u+2 v+w$, and has an underlying bi-grading $p=v+w, q=$ $u+v$, so that $W^{p, q}(A, \nabla)$ is the sum over all $u, v$ and $w$ satisfying these equations. The connection $\nabla$ will be used to define a differential on $W(A, \nabla)$. This will be a total differential

$$
d_{\nabla}=d_{\nabla}^{\mathrm{hor}}+d_{\nabla}^{\mathrm{ver}}
$$

where, to define $d_{\nabla}^{\text {hor }}$ and $d_{\nabla}^{\text {ver }}$ we look at $W(A, \nabla)$ from two different points of view.

First of all remark that, according to our conventions, the symmetric powers of the dual of the graded vector bundle $\mathcal{D}=A \oplus A$ (concentrated in degrees 0 and 1) has

$$
S^{p} \mathcal{D}^{*}=(\underbrace{\Lambda^{p} A^{*}}_{\text {degree }-p}) \oplus(\underbrace{A^{*} \otimes \Lambda^{p-1} A^{*}}_{\text {degree }-p+1}) \oplus \ldots \oplus(\underbrace{S^{p-1} A^{*} \otimes A^{*}}_{\text {degree }-1}) \oplus(\underbrace{S^{p} A^{*}}_{\text {degree } 0})
$$

hence

$$
W^{p, q}(A, \nabla)=\Omega\left(M ; S^{p} \mathcal{D}^{*}\right)^{q-p}
$$

We now use the representation up to homotopy structure induced by $\nabla$ on the double $\mathcal{D}$ of $A$ (see Example 3.8), which we dualize and extend to $S \mathcal{D}^{*}$. The resulting structure operator will be our vertical differential

$$
d_{\nabla}^{\mathrm{ver}}: W^{p, q}(A, \nabla) \rightarrow W^{p, q+1}(A, \nabla) .
$$

Similarly, for the coadjoint complex $\mathrm{Ad}^{*}$ one has

$$
S^{q} \mathrm{Ad}^{*}=(\underbrace{\Lambda^{q} T^{*} M}_{\text {degree }-q}) \oplus(\underbrace{A^{*} \otimes \Lambda^{q-1} T^{*} M}_{\text {degree }-q+1}) \oplus \ldots \oplus(\underbrace{T^{*} M \otimes S^{q-1} A^{*}}_{\text {degree }-1}) \oplus(\underbrace{S^{q} A^{*}}_{\text {degree } 0}),
$$

hence

$$
W^{p, q}(A, \nabla)=\Omega\left(A ; S^{q} \mathrm{Ad}^{*}\right)^{p-q} .
$$

We now use the connection $\nabla$ to form the coadjoint representation $\mathrm{Ad}_{\nabla}^{*}$. To obtain a horizontal operator which commutes with the vertical one, we consider the conjugation of the coadjoint representation, i.e. $\mathrm{Ad}^{*}$ with the structure operator $-\rho^{*}+\left(\nabla^{\mathrm{bas}}\right)^{*}+\left(R_{\nabla}^{\mathrm{bas}}\right)^{*}$. The symmetric powers will inherit a structure operator, and this will be our horizontal differential

$$
d_{\nabla}^{\text {hor }}: W^{p, q}(A, \nabla) \rightarrow W^{p+1, q}(A, \nabla) .
$$

Proposition 4.1. Endowed with $d_{\nabla}^{\text {hor }}$ and $d_{\nabla}^{v e r}, W(A, \nabla)$ becomes a differential bi-graded algebra whose cohomology is isomorphic to the cohomology of $M$. Moreover, up to isomorphisms of differential bi-graded algebras, $W(A, \nabla)$ does not depend on the choice of the connection $\nabla$.

Proof. To prove that the two differentials commute, one first remarks that it suffices to check the commutation relation on functions and on sections of $T^{*} M, \Lambda^{1} A^{*}$ and $S^{1} A^{*}$, which generate the entire algebra. This follows by direct computation (one can also use the local formulas below, but the computations are much more involved). The independence of $\nabla$ will follow from the intrinsic description of the Weil algebra, discussed in the next subsection. Finally, we need to prove that the cohomology equals that of $M$. Note that for $p>0$ the column $\left(W^{p, \bullet}, d_{\nabla}^{\mathrm{ver}}\right)$ is acyclic, since it corresponds to an acyclic representation up to homotopy of $T M$. Next, we note that the first column $\left(W^{0, \bullet}, d_{\nabla}^{\text {ver }}\right)$ is the De-Rham complex of $M$ and the usual spectral sequence argument provides the desired result. 
Remark 4.2 (Local coordinates). Since the operators are local, it is worth looking at their expressions in coordinates. Let us assume that we are over a chart $\left(x_{a}\right)$ of $M$ on which we have a trivialization $\left(e_{i}\right)$ of $A$. Over this chart, the Weil algebra will be the bigraded commutative algebras over the space of smooth functions, generated by elements $\partial^{a}$ of bidegree $(0,1)$ (1-forms), elements $\theta^{i}$ of bi-degree $(1,0)$ (the dual basis of $\left(e_{i}\right)$, viewed in $\Lambda^{1} A^{*}$ ), and elements $\mu^{i}$ of bi-degree $(1,1)$ (the dual basis of $\left(e_{i}\right)$, viewed in $\left.S^{1} A^{*}\right)$. A careful but straightforward computation shows that, on these elements,

$$
\begin{aligned}
d_{\nabla}^{\mathrm{ver}}\left(\partial^{a}\right) & =0 \\
d_{\nabla}^{\mathrm{ver}}\left(\theta^{i}\right) & =\mu^{i}-\Gamma_{a j}^{i} \partial^{a} \theta^{j} \\
d_{\nabla}^{\mathrm{ver}}\left(\mu^{i}\right) & =-\Gamma_{a j}^{i} \partial^{a} \mu^{j}+\frac{1}{2} r_{a b j}^{i} \partial^{a} \partial^{b} \theta^{j} \\
d_{\nabla}^{\mathrm{hor}}\left(\partial^{a}\right) & =-\rho_{i}^{a} \mu^{i}+\left(\frac{\partial \rho_{i}^{a}}{\partial x_{b}}-\Gamma_{b i}^{j} \rho_{j}^{a}\right) \theta^{i} \partial^{b}, \\
d_{\nabla}^{\mathrm{hor}}\left(\theta^{i}\right) & =-\frac{1}{2} c_{j k}^{i} \theta^{j} \theta^{k} \\
d_{\nabla}^{\mathrm{hor}}\left(\mu^{i}\right) & =-\left(c_{j k}^{i}+\sum_{a} \rho_{k}^{a} \Gamma_{a j}^{i}\right) \theta^{j} \mu^{k}+\frac{1}{2} R_{j k a}^{i} \theta^{j} \theta^{k} \partial^{a} .
\end{aligned}
$$

Here we use the Einstein summation convention. $\rho_{i}^{a}$ are the coefficients of $\rho, c_{j k}^{i}$ are the structure functions of $A, r_{a b j}^{i}$ are the coefficients of the curvature of $\nabla$ and $R_{j k a}^{i}$ are the coefficients of the basic curvature:

$$
\begin{gathered}
\rho\left(e_{i}\right)=\sum \rho_{i}^{a} \partial_{a}, \quad\left[e_{j}, e_{k}\right]=\sum c_{j k}^{i} e_{i}, \\
R_{\nabla}\left(\partial_{a}, \partial_{b}\right) e_{j}=r_{a b j}^{i} e_{i}, \quad R_{\nabla}^{\mathrm{bas}}\left(e_{j}, e_{k}\right) \partial_{a}=R_{j k a}^{l} e_{l} .
\end{gathered}
$$

Note that for a smooth function $f$,

$$
d_{\nabla}^{\mathrm{ver}}(f)=\partial_{a}(f) \partial^{a}, \quad d_{\nabla}^{\mathrm{hor}}(f)=\partial_{a}(f) \rho_{i}^{a} \omega^{i} .
$$

Also, in case that the connection $\nabla$ is flat, all the $\Gamma$ and $r$-terms above vanish, while the $R$-terms are given by the partial derivatives of the structure functions $c_{j k}^{i}$.

Example 4.3 (The standard Weil algebra). In the case of Lie algebras $\mathfrak{g}$, one can immediately see that we recover the standard Weil algebra $W(\mathfrak{g})$. In particular, the local coordinates description becomes

$$
\begin{aligned}
d^{\mathrm{ver}}\left(\theta^{i}\right) & =\mu^{i}, \\
d^{\mathrm{ver}}\left(\mu^{i}\right) & =0, \\
d^{\mathrm{hor}}\left(\theta^{i}\right) & =-\frac{1}{2} \sum_{j, k} c_{j k}^{i} \theta^{j} \theta^{k}, \\
d^{\mathrm{hor}}\left(\mu^{i}\right) & =-\sum_{j, k} c_{j k}^{i} \theta^{j} \mu^{k},
\end{aligned}
$$

which is the standard description of the Weil algebra. In this case, $d^{\mathrm{ver}}$ is usually called the Koszul differential, denoted $d_{K}, d^{\text {hor }}$ is called the Cartan differential, denoted $d_{C}$, and the total differential is denoted by $d_{W}$.

Example 4.4 (The BRST algebra). Recall Kalkman's BRST algebra [19] associated to a $\mathfrak{g}$-manifold $M$. It is $W(\mathfrak{g}, M):=W(\mathfrak{g}) \otimes \Omega(M)$ with differential:

$$
\delta=d_{W} \otimes 1+1 \otimes d_{D R}+\sum_{a=1}^{n} \theta^{a} \otimes \mathcal{L}_{a}-\sum_{b=1}^{n} \mu^{b} \otimes \iota_{b} .
$$


Proposition 4.5. Let $A=\mathfrak{g} \ltimes M$ be the action algebroid associated to a $\mathfrak{g}$-manifold $M$. Then

$$
W(\mathfrak{g}, M)=W\left(A ; \nabla^{f l a t}\right),
$$

where $\nabla^{\text {flat }}$ is the canonical flat connection on $A$.

Proof. Follows immediately from the local coordinates description of the differentials of the Weil algebra.

\subsection{The intrinsic model}

In this subsection we describe an intrinsic version of the Weil algebra, which we will denote $W(A)$. An element $c \in W^{p, q}(A)$ is a string $c=\left(c_{0}, c_{1}, \ldots\right)$, where each of the components $c_{i}$ is an antisymmetric multilinear map

$$
c_{i}: \underbrace{\Gamma(A) \times \ldots \times \Gamma(A)}_{(p-i) \text { times }} \rightarrow \Omega^{q-i}\left(M ; S^{i}\left(A^{*}\right)\right)
$$

and they are related by:

$$
c_{i}\left(\alpha_{1}, \ldots, f \alpha_{p-i}\right)=f c_{i}\left(\alpha_{1}, \ldots, f \alpha_{p-i}\right)-d f \wedge c_{i+1}\left(\alpha_{1}, \ldots, \alpha_{p-i-1}\right) \alpha_{p} .
$$

As in Example 3.26, one should think of $c_{1}, c_{2}, \ldots$ as correction terms that control the lack of $C^{\infty}(M)$-linearity of $c_{0}$. The horizontal differential $d^{\text {hor }}$ (which increases $p$ ) is defined as follows.

$$
\begin{aligned}
\left(d^{\text {hor }} c\right)_{0}\left(\alpha_{1}, \ldots, \alpha_{p+1}\right)= & \sum_{i<j}(-1)^{i+j} c_{0}\left(\left[\alpha_{i}, \alpha_{j}\right], \ldots, \hat{\alpha_{i}}, \ldots, \hat{\alpha_{j}}, \ldots, \alpha_{p+1}\right)+ \\
& +\sum_{i}(-1)^{i+1} L_{\rho\left(\alpha_{i}\right)}\left(c_{0}\left(\alpha_{1}, \ldots, \hat{\alpha_{i}}, \ldots, \alpha_{p+1}\right)\right)
\end{aligned}
$$

while the other terms can be computed using the compatibility between the components (see Example 3.26). The vertical differential $d^{\mathrm{ver}}$ (which increases $q$ ) is simpler: $\left(d^{\mathrm{ver}} c\right)_{0}$ is, up to a sign, just the De-Rham differential

$$
\left(d^{\mathrm{ver}} c\right)_{0}\left(\alpha_{1}, \ldots, \alpha_{p}\right)=(-1)^{p} d_{\mathrm{DR}}\left(c_{0}\left(\alpha_{1}, \ldots, \alpha_{p}\right)\right),
$$

again, the higher order terms can be computed from the relation above.

Theorem 4.6. Endowed with $d^{\text {hor }}$ and $d^{v e r}, W(A)$ becomes a differential bi-graded algebra. Moreover, for any connection $\nabla$ on $A, W(A)$ is naturally isomorphic to $W(A, \nabla)$.

Proof. Let us describe the isomorphism $\phi_{\nabla}: W(A) \rightarrow W(A, \nabla)$ on generators of low degree. First we observe that $W^{1,0}(A) \cong \Omega^{1}(A) \cong W^{1,0}(A, \nabla)$ and $W^{0,1}(A) \cong \Omega^{1}(M) \cong W^{0,1}(A, \nabla)$, so we can define the operator to be the identity in those degrees. For $c=\left(c_{0}, c_{1}\right) \in W^{1,1}(A)$ we set

$$
\phi_{\nabla} c=\left(\phi_{\nabla} c\right)_{0}+\left(\phi_{\nabla} c\right)_{1} \in \Omega^{1}(A, M) \otimes \Gamma\left(S^{1}\left(A^{*}\right)\right),
$$

where

$$
\begin{aligned}
\left(\phi_{\nabla} c\right)_{0}(\alpha)(X) & =c_{1}\left(\nabla_{X}(\alpha)\right)-c_{0}(\alpha)(X) \\
\left(\phi_{\nabla} c\right)_{1} & =c_{1}
\end{aligned}
$$

for $\alpha \in \Gamma(A)$ and $X$ a vector field on $M$. The map $\phi_{\nabla}$ is an isomorphism since its inverse can be easily computed in terms of the generators. Finally, the fact that the differentials coincide can be checked in low degree. 
Example 4.7 (IM forms). We now show that the equations underlying the notion of IM form of an algebroid (see Remark 2.7) are cocycle conditions in the Weil algebra.

Proposition 4.8. Given a Lie algebroid A, there is a 1-1 correspondence between IM forms $\sigma$ on $A$ and elements $c \in W^{1,2}(A)$ which are both $d^{\text {hor }}$-closed and $d^{\text {ver }}$-closed.

Proof. The columns of the Weil algebra are exact and the fact that $d^{\mathrm{ver}}(c)=0$ implies that there exists a unique $c^{\prime} \in W^{1,1}(A)$ of the form $c^{\prime}=(\sigma, 0)$ such that $c=d^{\text {ver }}\left(c^{\prime}\right)$. An element in $W^{2,2}(A)$ has three components, therefore, we obtain three equations:

$$
d^{\text {hor }}(c)_{0}=d^{\text {hor }}(c)_{1}=d^{\text {hor }}(c)_{2}=0 .
$$

It is easy to check that, because $c=d^{\mathrm{ver}}\left(c^{\prime}\right)$, the first equation follows from the other two. In terms of $\sigma$, the last two equations are:

$$
\begin{gathered}
d^{\text {hor }}(c)_{1}(\alpha)(\beta)=-\sigma[\alpha, \beta]+L_{\rho(\alpha)}(\sigma(\beta))-L_{\rho(\beta)}(\sigma(\alpha))-d\langle\sigma(\alpha), \rho(\beta)\rangle=0, \\
d^{\text {hor }}(c)_{2}(\alpha, \beta)=-i_{\rho(\alpha)}(\sigma(\beta))-i_{\rho(\beta)}(\sigma(\alpha))=0 .
\end{gathered}
$$

These are precisely the equations appearing in the definition of IM forms.

In a coming paper [3] we will show that the Weil algebra comes together with a Van Est map, from which we will deduce the main result of [7] which establishes a 1-1 correspondence between closed multiplicative 2-forms on groupoids and IM forms on the underlying algebroids.

\section{References}

[1] Jesús A. Alvarez López. A decomposition theorem for the spectral sequence of Lie foliations. Trans. Amer. Math. Soc., 329(1):173-184, 1992.

[2] C. Arias Abad and M. Crainic. Representations up to homotopy of groupoids and the Bott spectral sequence. In preparation.

[3] C. Arias Abad and M. Crainic. The Weil algebra and Van Est isomorphisms. Preprint 2008.

[4] A. Blaom. Lie algebroids and Cartan's method of equivalence. Arxiv math/0509071.

[5] R. Bott. On the Chern-Weil homomorphism and the continuous cohomology of Liegroups. Advances in Math., 11:289-303, 1973.

[6] Raoul Bott. Lectures on characteristic classes and foliations. In Lectures on algebraic and differential topology (Second Latin American School in Math., Mexico City, 1971), pages 1-94. Lecture Notes in Math., Vol. 279. Springer, Berlin, 1972. Notes by Lawrence Conlon, with two appendices by J. Stasheff.

[7] Henrique Bursztyn, Marius Crainic, Alan Weinstein, and Chenchang Zhu. Integration of twisted Dirac brackets. Duke Math. J., 123(3):549-607, 2004.

[8] Henri Cartan. Notions d'algèbre différentielle; application aux groupes de Lie et aux variétés où opère un groupe de Lie. In Colloque de topologie (espaces fibrés), Bruxelles, 1950, pages 15-27. Georges Thone, Liège, 1951.

[9] M. Crainic. On the perturbation lemma and deformations. Arxiv math/0403266.

[10] M. Crainic and R. L. Fernandes. Stability of symplectic leaves. Arxiv math/0810.4437.

[11] M. Crainic and R. L. Fernandes. Secondary characteristic classes of Lie algebroids. In Quantum field theory and noncommutative geometry, volume 662 of Lecture Notes in Phys., pages 157-176. Springer, Berlin, 2005. 
[12] M. Crainic and I. Moerdijk. Deformations of Lie brackets: cohomological aspects. J. Eur. Math. Soc., 10:1037-1059, 2008.

[13] Marius Crainic and Rui Loja Fernandes. Integrability of Lie brackets. Ann. of Math. (2), 157(2):575-620, 2003.

[14] Sam Evens, Jiang-Hua Lu, and Alan Weinstein. Transverse measures, the modular class and a cohomology pairing for Lie algebroids. Quart. J. Math. Oxford Ser. (2), 50(200):417-436, 1999.

[15] Ezra Getzler. The equivariant Chern character for non-compact Lie groups. Adv. Math., 109(1):88-107, 1994.

[16] James L. Heitsch. A cohomology for foliated manifolds. Comment. Math. Helv., 50:197$218,1975$.

[17] Dale Husemoller, John C. Moore, and James Stasheff. Differential homological algebra and homogeneous spaces. J. Pure Appl. Algebra, 5:113-185, 1974.

[18] D. Iglesias-Ponte, C. L. Gengoux, and P. Xu. Universal lifting theorem and quasi-Poisson groupoids. Arxiv math/0507396.

[19] Jaap Kalkman. BRST model for equivariant cohomology and representatives for the equivariant Thom class. Comm. Math. Phys., 153(3):447-463, 1993.

[20] Franz W. Kamber and Philippe Tondeur. Foliations and metrics. In Differential geometry (College Park, Md., 1981/1982), volume 32 of Progr. Math., pages 103-152. Birkhäuser Boston, Boston, MA, 1983.

[21] Y. Kosmann-Schwarzbach and K. C. H. Mackenzie. Differential operators and actions of Lie algebroids. In Quantization, Poisson brackets and beyond (Manchester, 2001), volume 315 of Contemp. Math., pages 213-233. Amer. Math. Soc., Providence, RI, 2002.

[22] K. Mackenzie. Lie groupoids and Lie algebroids in differential geometry, volume 124 of London Mathematical Society Lecture Note Series. Cambridge University Press, Cambridge, 1987.

[23] K. Mackenzie and P. Higgins. Algebraic constructions in the category of Lie algebroids. J. Algebra, 129:194-230, 1990.

[24] R. Mehta. Q-algebroids and their cohomology. Arxiv math/0703234.

[25] Daniel Quillen. Superconnections and the Chern character. Topology, 24(1):89-95, 1985.

[26] Stefan Schwede. Morita theory in abelian, derived and stable model categories. In Structured ring spectra, volume 315 of London Math. Soc. Lecture Note Ser., pages 33-86. Cambridge Univ. Press, Cambridge, 2004.

[27] Jim Stasheff. Differential graded Lie algebras, quasi-Hopf algebras and higher homotopy algebras. In Quantum groups (Leningrad, 1990), volume 1510 of Lecture Notes in Math., pages 120-137. Springer, Berlin, 1992.

[28] Charles A. Weibel. An introduction to homological algebra, volume 38 of Cambridge Studies in Advanced Mathematics. Cambridge University Press, Cambridge, 1994. 